ARTICLE

\title{
Blockade of the AHR restricts a Treg-macrophage suppressive axis induced by L-Kynurenine
}

Luis Felipe Campesato 1,2, Sadna Budhu (D) 1,2, Jeremy Tchaicha ${ }^{3}$, Chien-Huan Weng (1) 1,2, Mathieu Gigoux (1) 1,2, Ivan Jose Cohen ${ }^{4}$, David Redmond ${ }^{1,2}$, Levi Mangarin¹,2, Stephane Pourpe ${ }^{1,2}$, Cailian Liu¹,2, Roberta Zappasodi (10 1,2, Dmitriy Zamarin 1,2, Jill Cavanaugh ${ }^{3}$, Alfredo C. Castro ${ }^{3}$, Mark G. Manfredi ${ }^{3}$, Karen McGovern ${ }^{3}$, Taha Merghoub (i) ${ }^{1,2,5 凶}$ \& Jedd D. Wolchok (i) $1,2,5 \bowtie$

Tryptophan catabolism by the enzymes indoleamine 2,3-dioxygenase 1 and tryptophan 2,3dioxygenase 2 (IDO/TDO) promotes immunosuppression across different cancer types. The tryptophan metabolite L-Kynurenine (Kyn) interacts with the ligand-activated transcription factor aryl hydrocarbon receptor (AHR) to drive the generation of Tregs and tolerogenic myeloid cells and PD-1 up-regulation in CD8 ${ }^{+} \mathrm{T}$ cells. Here, we show that the AHR pathway is selectively active in IDO/TDO-overexpressing tumors and is associated with resistance to immune checkpoint inhibitors. We demonstrate that IDO-Kyn-AHR-mediated immunosuppression depends on an interplay between Tregs and tumor-associated macrophages, which can be reversed by AHR inhibition. Selective AHR blockade delays progression in IDO/TDOoverexpressing tumors, and its efficacy is improved in combination with PD-1 blockade. Our findings suggest that blocking the AHR pathway in IDO/TDO expressing tumors would overcome the limitation of single IDO or TDO targeting agents and constitutes a personalized approach to immunotherapy, particularly in combination with immune checkpoint inhibitors.

\footnotetext{
${ }^{1}$ Swim Across America and Ludwig Collaborative Laboratory, Immunology Program, Parker Institute for Cancer Immunotherapy, Memorial Sloan Kettering Cancer Center, New York, NY, USA. ${ }^{2}$ Immuno-Oncology Service, Human Oncology and Pathogenesis Program, Memorial Sloan Kettering Cancer Center, New York, NY, USA. ${ }^{3}$ Ikena Oncology, Boston, MA, USA. ${ }^{4}$ Department of Neurology and Louis V. Gerstner Jr Graduate School of Biomedical Sciences, Memorial Sloan Kettering Cancer Center, New York, NY 10065, USA. ${ }^{5}$ These authors contributed equally: Taha Merghoub, Jedd D. Wolchok.

凶email: merghout@mskcc.org; wolchokj@mskcc.org
} 
$\mathrm{T}$ he catabolism of the essential amino acid Tryptophan (Trp) into Kynurenine (Kyn) metabolites is a central pathway maintaining peripheral tolerance to immuneprivileged sites (eye, testis, and placenta), together with the adenosine/purinergic pathway and with immune checkpoints, such as CTLA-4 and PD- $1^{1}$. Expression of indoleamine 2,3-dioxygenase 1 (IDO1), or tryptophan 2,3-dioxygenase 2 (TDO2)hereafter referred to IDO/TDO-by tumors has been shown to act as a driver of immune suppression through myeloid-derived suppressor cell (MDSCs) recruitment and activation ${ }^{2}$, T-cell anergy $^{3}$ and is associated with poor clinical prognosis in glioblastoma ${ }^{4}$ or acquired resistance mechanism to PD-1 and CTLA4 blockade in pre-clinical models $2,5,6$. Several efforts have been directed to blocking the IDO pathway. More recently the inhibition of IDO pathway among unselected patient populations in a large Phase III trial (ECHO-301/KEYNOTE-252) did not improve the therapeutic outcome to PD-1 blockade 7 , but highlight the need for further studies aimed at investigating the mechanisms of metabolic dysregulation via Trp catabolism in antitumor immunity.

Recent research has revealed that the IDO/TDO product Kyn can act as a key signaling molecule through activation of the ligand-activated transcription factor aryl hydrocarbon receptor (AHR), which is implicated in a variety of biochemical processes including control of the immune response ${ }^{8-10}$. The production of Kyn and its metabolites by TDO was shown to reach concentrations sufficient to activate the AHR pathway in the tumor microenvironment (TME) of a glioblastoma model ${ }^{4}$. Recent studies demonstrated that Kyn can be found in high concentrations in the plasma of advanced-stage cancer patients and a high serum Kyn/Trp ratio correlates with poor prognosis after PD-1 blockade in several cancer types, including lung cancer, melanoma, and renal cell carcinomas ${ }^{11-13}$, while systemic Kyn depletion with a PEGylated Kyn-degrading enzyme led to cancer control in pre-clinical settings ${ }^{14}$. In the present study, we identify AHR pathway activity, a downstream component of IDO/TDOKyn axis, as a common feature of cancers expressing IDO or TDO and as a driver of T cell dysfunction by promoting a Tregmacrophage suppressive axis in the TME. We show that targeting the AHR in tumors with an active Trp catabolic pathway overcomes immunosuppressive mechanisms and sensitize tumors to anti-PD-1 therapy. Our findings provide a rationale for assessing active metabolic pathways in tumors, in a precision-medicine type of approach, and exploring AHR inhibition in combination with other immunotherapy strategies.

\section{Results}

AHR expression is associated with immunosuppression in human tumors. To address whether the IDO/TDO-Kyn-AHR axis limits the efficacy of immune-based therapies, we examined an RNAseq dataset of tumors from 68 patients with advanced melanoma before and after anti-PD-1 therapy ${ }^{15}$. We found an enrichment in the expression of enzymes involved in Kyn degradation, such as kynurenine 3-monooxygenase (KMO), kynureninase (KYNU) and 3-Hydroxyanthranilic acid dioxygenase $(H A A O)$, in patients who derived clinical benefit from PD-1 blockade (Fig. 1a) in comparison to those who did not $(P=$ $0.001)$. We further investigated the potential role of AHR in promoting well-defined immune suppressive mechanisms. Using single-cell suspensions prepared from malignant melanoma resections banked at MSK and classified as low or high based on their intracellular expression of IDO (Fig. 1b), we detected higher levels of immune-regulatory factors ( $I L-10, P D-L 1$, and CTLA-4) in IDO ${ }^{\text {high }}$ samples compared to IDOlow samples (Fig. 1c). Furthermore, we detected higher levels of the AHR-target genes cytochrome P4501A1 (CYP1A1) and cytochrome P4501B1 (CYP1B1) in IDO ${ }^{\text {high }}$ samples versus IDO $^{\text {low }}$ samples. Increases in the AHR-target genes were reversed upon treatment with a selective AHR inhibitor in vitro (KYN-101) (Fig. 1d).

Using the expression of CYP1B1 as a surrogate of AHR activity, we found its association with poorer overall survival in renal and urothelial cancer patients (Supplementary Fig. 1a). In addition, CYP1B1 expression was positively correlated with both IDO1 and TDO2 expression in skin melanoma (SKCM), squamous lung carcinoma (LUSC), diffuse large B-cell lymphoma (DLBC) and pancreatic adenocarcinoma (PDAC) (Supplementary Fig. 1b). These obeservations suggest the existence of IDO/TDO-mediated activation of the AHR pathway across cancer types. Using a recently described classification of tumors based on their immunogenomic profile ${ }^{16}$, we found an upregulated expression of Kyn-AHR pathway-related genes (TDO2, AHR, and CYP1B1) within tumors presenting a TGF- $\beta$-dominant immune signature (C6 in Fig. 1e). Such signature has been reported as the one predicting the poorest prognosis and associated with a myeloid dominated microenvironment, with low lymphocytic infiltrate and high on M2-TAMs content on their constituent tumors ${ }^{16}$. Expression of TDO2, $A H R$, and CYP1B1 was the highest in the TGF- $\beta$-dominant immune subtype (C6), while IDO1 was found highest in the interferon gamma (IFN- $\gamma$ ) dominant signature (C2) followed by C6, likely in reflection to its IFN-inducible nature ${ }^{17}$. Furthermore, TCGA RNAseq analysis revealed a strong correlation between AHR-pathway related genes (IDO1, TDO2, and CYP1B1) and myeloid-cell markers (MRC1/CD206, CD14), Treg markers (FOXP3, IL2RA) and inhibitory checkpoints (PDCD1, CTLA-4) (Fig. 1e and Supplementary Fig. 1c). Taken together, these findings suggest that activation of the Kyn-AHR pathway is a common feature of IDO or TDO-expressing cancers and may provide a novel immunoregulatory mechanism associated with resistance to checkpoint inhibition.

Immune dysfunction is a feature of IDO/TDO-expressing tumors. To further investigate the role of the Kyn-AHR pathway in promoting cancer-associated immune suppression in a more controlled setting, we generated a pre-clinical model of B16-F10 melanoma overexpressing IDO (B16 $\left.{ }^{\mathrm{IDO}}\right)$ or TDO $\left(\mathrm{B} 16^{\mathrm{TDO}}\right)$. These models allowed us to isolate the effects of IDO or TDO expression by comparing it to the parental cell line $\left(\mathrm{B} 16^{\mathrm{WT}}\right)$, which expresses minimal levels of the two enzymes. Increased production of Kyn was detected in $\mathrm{B} 16^{\mathrm{IDO}}$ and $\mathrm{B} 16^{\mathrm{TDO}}$ compared to B16 ${ }^{\mathrm{WT}}$ (Supplementary Fig. 2a). We found no differences in the tumor growth rate of $\mathrm{B} 16^{\mathrm{IDO}}$ and $\mathrm{B} 16^{\mathrm{TDO}}$ tumors (Supplementary Fig. 2b) when implanted in immunodeficient $\mathrm{Rag}^{-1-}$ or WT immunocompetent mice. In contrast, a faster tumor growth is observed in B16 ${ }^{\mathrm{WT}}$ tumors in $\mathrm{Rag}^{-1-}$ comparison to WT. These finding suggest that $\mathrm{T}$ cells play a role at controlling tumor progression in the $\mathrm{B} 16^{\mathrm{WT}}$ models, but not $\mathrm{B} 16^{\mathrm{IDO} / \mathrm{TDO}}$. We hypothesized that an immunosuppressive TME induced by IDO/TDO overexpression could promote a dysfunctional $\mathrm{T}$ cell effector function. Therefore, we next investigated the extent of altered tumor immunity in the context of Kyn-AHR pathway activation to further delineate the mechanisms underlying IDO-induced immune suppression using the $\mathrm{B} 16^{\mathrm{IDO}}$ model. Gene expression analysis of whole-tumors revealed a distinct immune signature in $\mathrm{B} 16^{\mathrm{IDO}}$ tumors, with reduced expression level of Th1 genes, including IFNG, IL1B, TNFA, CD40, CD86 and GZMB (Supplementary Fig. 2c). Multiplex analysis of intratumoral cytokines revealed reduced levels of T cell-related cytokines, such as IFN $\gamma$, CXCL9, CCL5/RANTES and IL-15, but increased VEGF, a known AHR-responsive gene ${ }^{18}$ (Supplementary Fig. 2d). To assess the tumoricidal functional state of intratumoral $\mathrm{CD}^{+} \mathrm{T}$ cells, we 
a
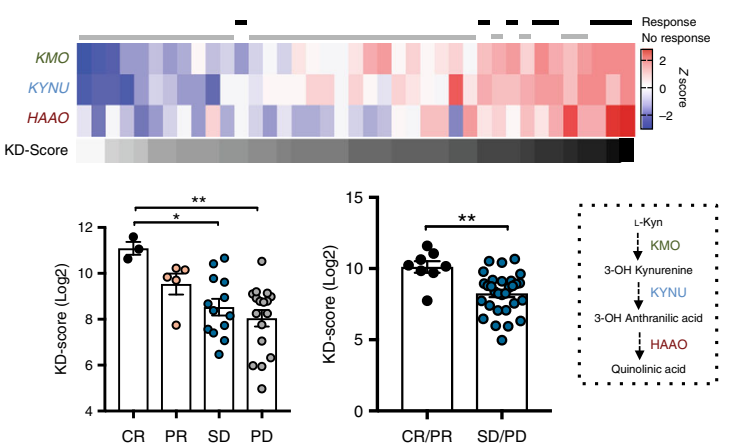

b
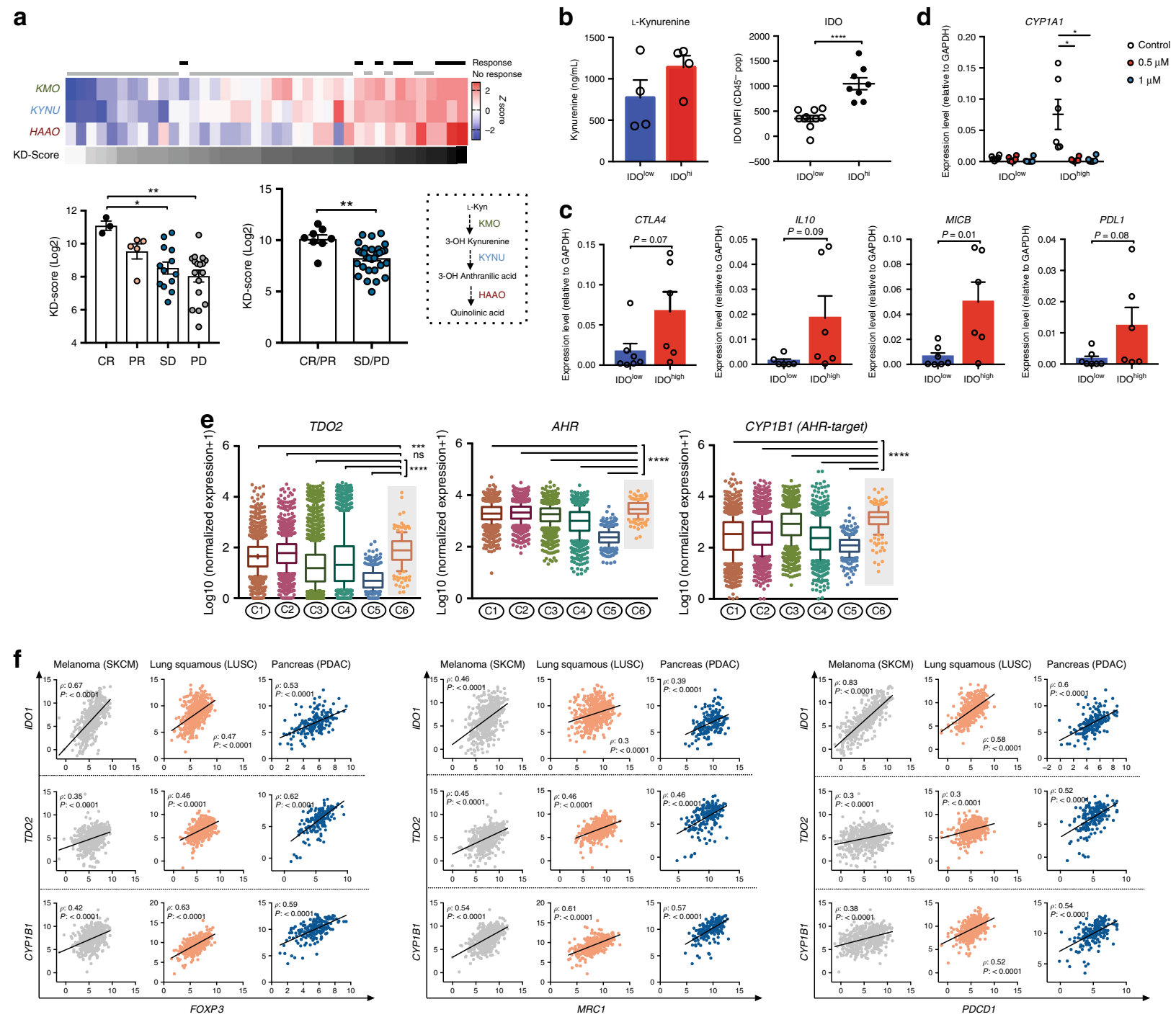

Fig. 1 An active IDO/TDO-Kyn-AHR pathway associates with immune suppressive features in human cancers. a Top: heat-map representing the geneexpression analysis of Kyn-degrading enzymes (KMO, KYNY, HAAO) and KD-Score (grayscale) in responsive (complete response/CR or partial response/ PR) highlighted in black $(n=8)$, or non responsive (stable disease/SD or progressive disease/PD) highlighted in gray $(n=31)$, melanoma patients after PD-1 blockade. Bottom: bar graph with quantification of KD-Score. $\mathbf{b}$ ELISA quantification of L-Kynurenine in blood serum of melanoma patients (left) categorized as IDOhigh or IDOlow ( $n=4$ IDO high and $n=4$ IDOlow) based on intracellular IDO staining by FACS of melanoma cell suspensions (right) ( $n=$ 8 IDO high and $n=10$ IDO $^{\text {low }}$ ). $\mathbf{c}$ mRNA of immunoregulatory markers by qRT-PCR analysis in IDOhigh and IDOlow melanoma cell suspensions. $\mathbf{d}$ mRNA of AHR-target genes CYP1A1 and CYP1B1 by GRT-PCR analysis in IDO ${ }^{\text {high }}$ and IDO ${ }^{\text {low }}$ melanoma cell suspensions after treatment with selective AHR inhibitor $\mathrm{KYN}-101$ for $24 \mathrm{~h}$ ( $n=6$ IDOhigh and $n=6-7$ IDOlow). e Distribution of log-transformed expression levels of AHR-related genes (TDO2, AHR, and CYP1B1) across six immune subtypes of cancer (C1: wound-healing, C2: IFN- $\gamma$ dominant, C3: inflammatory, C4: lymphocyte-depleted, C5: immunologically quiet, C6: TGF- $\beta$ dominant). Data plotted as box and whiskers with the median and limits within the $10-90 \%$ percentile. $\mathbf{f}$ Correlation analysis between Treg marker (FOXP3), myeloid-cell marker (MRC1/CD206), inhibitory checkpoint (PDCD1/PD-1), and AHR-related genes IDO1, TDO2, and CYP1B1 in TCGA RNAseq data of skin melanoma (SKCM), squamous lung (LUSC) and pancreatic adenocarcinoma (PDAC) analyzed by Spearman rank correlation. Data shown are represented as mean values \pm SEM with two-tailed unpaired Student's $t$ test in (a-d), one-way ANOVA test with Tukey correction in (a) and Kruskal-Wallis with Dunn correction in (e). $P$ value: ${ }^{\star} P<0.05,{ }^{\star \star} P<0.01,{ }^{\star \star \star} P<0.001,{ }^{\star \star \star \star} P<0.0001$.

sorted $\mathrm{CD}^{+} \mathrm{T}$ cells from IDO-overexpressing tumors by fluorescence activated cell sorting (FACS) and further assessed them in a 3D collagen-fibrin-based killing assay, as previously described ${ }^{19}$. Analysis of the killing ability of effector $\mathrm{CD}^{+} \mathrm{T}$ cells revealed a dysfunctional phenotype, with a $30 \%$ killing efficiency of $\mathrm{B} 16^{\mathrm{IDO}}$. isolated $\mathrm{CD} 8{ }^{+} \mathrm{T}$ cells as compared to $54 \%$ in $\mathrm{B} 16^{\mathrm{WT}}$-isolated $\mathrm{CD}^{+} \mathrm{T}$ cells $(P=0.01)$ and $70 \%$ of control in vitro activated tumor antigen-specific $\mathrm{CD} 8^{+} \mathrm{T}$ cells (pmel1.1), which recognize the melanosomal tumor antigen gp100 (pmel1.1) $(P=0.009)$ (Fig. 2a). In addition, supernatants from $\mathrm{CD}^{+} \mathrm{T}$ cells isolated from $\mathrm{B} 16^{\mathrm{IDO}}$ tumors contained lower levels of effector $\mathrm{T}$ cell cytokines, including IFN $\gamma$, IL-1 $\beta$, RANTES, and TNFa after restimulation with anti-CD3/CD28 as compared to $\mathrm{B} 16^{\mathrm{WT}}$-isolated $\mathrm{CD}^{+} \mathrm{T}$ cells (Fig. 2b). Similar dysfunctional phenotype was observed in $\mathrm{CD}^{+}{ }^{+} \mathrm{T}$ cells of the $\mathrm{B} 16^{\mathrm{TDO}}$ model (Supplementary Fig. 2e) Taken together, our results support a causal role for the expression of Trp-catabolic enzymes in driving an adaptive immune resistance mechanism, in part through inhibition of $\mathrm{CD}^{+} \mathrm{T}$ cells cytolytic function.

Further analysis of the immune-infiltrate in $\mathrm{B} 16^{\mathrm{IDO}}$ tumors revealed an enrichment of myeloid cells, with increased abundance of TAMs $\left(\mathrm{CD} 11 \mathrm{~b}^{+} \mathrm{F} 4 / 80^{\text {high }} \mathrm{Ly}_{6 \mathrm{G}}{ }^{-}\right)$and $\mathrm{CD} 11 \mathrm{c}^{+}$ 

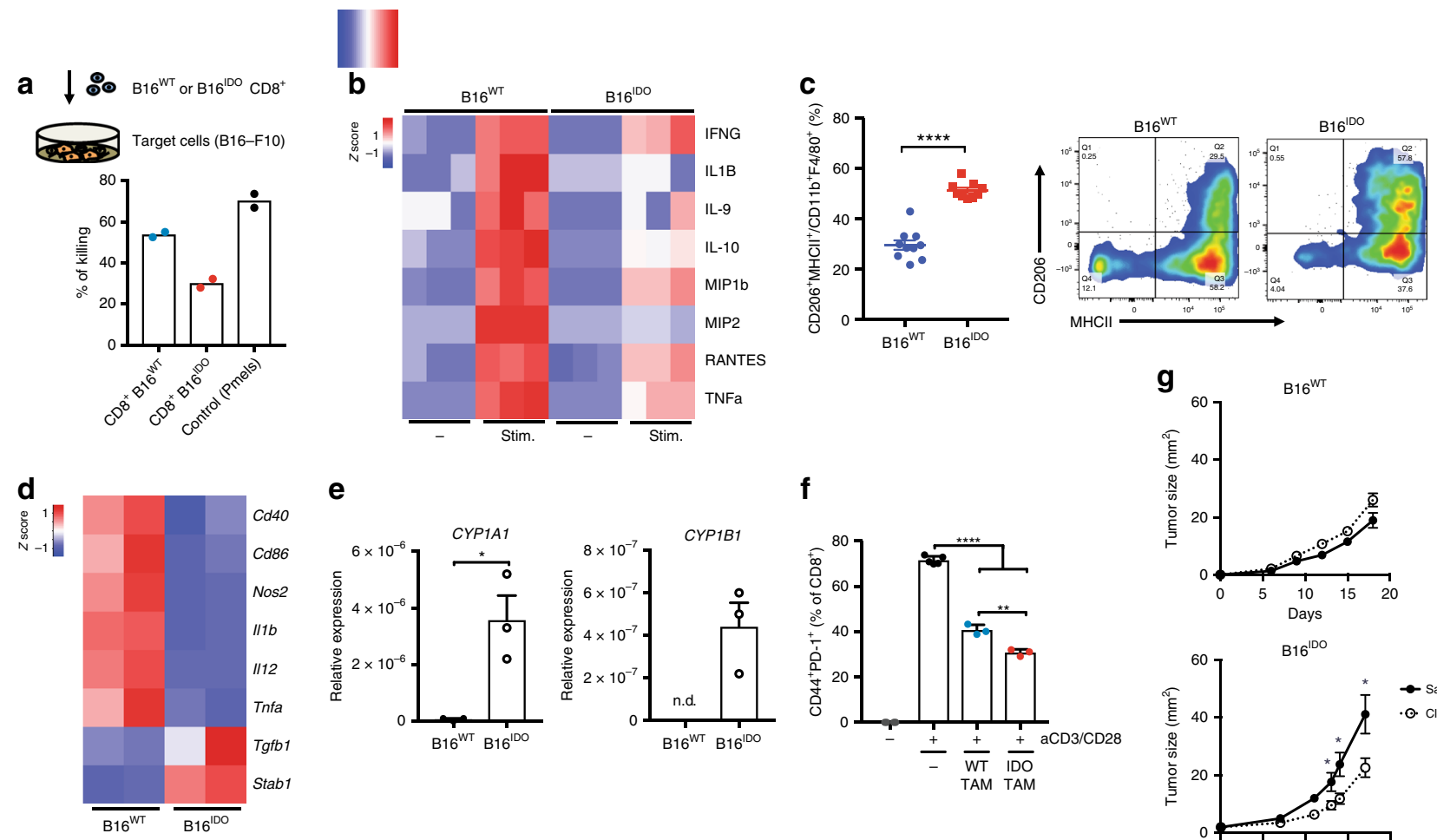

$\mathbf{e}$

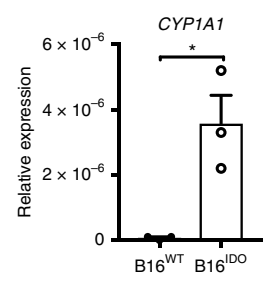

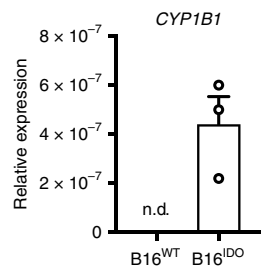

f

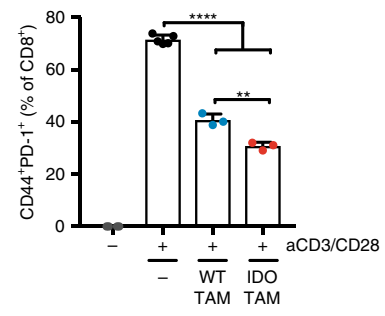

g
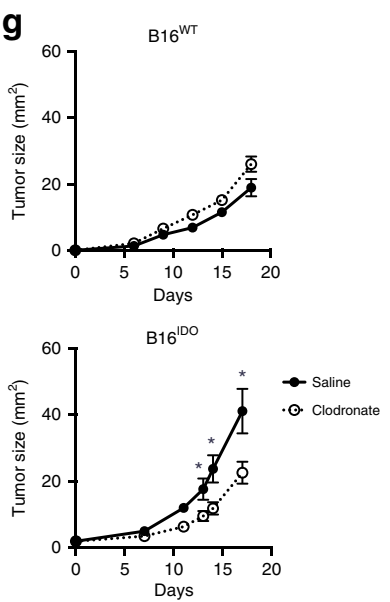

Fig. 2 IDO-overexpressing melanomas present a dysfunctional myeloid-enriched immune landscape. a Top: schematic representation of the experimental setup for the 3D collagen-fibrin gel killing assay and bottom, quantification of target cell killing by tumor-isolated CD8 ${ }^{+} \mathrm{T}_{\text {cells }}$ or tumorantigen (gp100)-specific CD8 ${ }^{+} \mathrm{T}$ cells (pmels) (Data is representative of two independent experiments). $\mathbf{b}$ Multiplex analysis of cytokines from ex vivo tumor-isolated $\mathrm{CD}^{+} \mathrm{T}$ cells stimulated with aCD3/CD28 beads for $48 \mathrm{~h}$. c, flow cytometric analysis and quantification of M2-like TAMs (CD206 ${ }^{+}$ $\mathrm{MHClI}^{+}$in $\left.\mathrm{CD} 11 \mathrm{~b}^{+} \mathrm{F} 4 / 80^{+}\right)$cell populations in B16 IDO or B16WT tumors at day 14 post implantation $\left(n=10\left(\mathrm{~B} 16^{\mathrm{WT}}\right)\right.$ and $n=9$ (B16 IDO)). Results are representative of three independent experiments. $\mathbf{d}$ Heat-map of mRNA expression of M1 and M2 markers and $\mathbf{e}$, of AHR-target genes in TAMs as determined by RT-PCR, data were relative to GAPDH expression and $z$-score normalized $(n=3)$. $\mathbf{f}$ in vitro suppressive activity of TAMs purified from B16 IDO or B16 WT tumor-bearing mice at day 14 post implantation in $\mathrm{CD}^{+}$T cell activation state at a ratio of $1: 2 \mathrm{CD} 8^{+}$to TAMs $(n=3)$. $\mathbf{g}$ Tumor progression of $\mathrm{B} 16^{\mathrm{IDO}}$ or $\mathrm{B} 16 \mathrm{WT}$ implanted in mice treated with clodronate liposome $(1 \mathrm{mg} / \mathrm{mouse})(n=8)$ or control $(n=8)$. Results are representative of two independent experiments. Data is represented as mean values \pm SEM. Two-tailed unpaired Student's $t$ test in (c-e, $\mathbf{g})$ and one-way ANOVA test with Tukey correction in (f). $P$ value: ${ }^{\star} P<0.05,{ }^{\star \star} P<0.01,{ }^{\star \star \star} P<0.001,{ }^{\star \star \star \star} P<0.0001$.

dendritic cells (DCs) $\left(\mathrm{CD} 11 \mathrm{c}^{+} \mathrm{MHCII}{ }^{+}\right)$in comparison with B16 WT tumors (Fig. 2c, Supplementary Fig. 3a). Similar phenotype was observed in the B16 ${ }^{\mathrm{TDO}}$ model (Supplementary Fig. 2e) and other cancer cell line models expressing high levels of IDO in baseline conditions, such as the breast carcinoma $4 \mathrm{~T}^{2}$ and colorectal cancer model MC $38^{20}$. Similar to a recent report linking the AHR with a suppressive state of TAMs in a glioblastoma model ${ }^{21}$, tumor cell overexpression of IDO promoted an immunoregulatory M2-like phenotype in TAMs. $\mathrm{B} 16^{\mathrm{IDO}}$ TAMs were more frequent and presented increased expression of the mannose receptor CD206/MRC1, an M2marker (Fig. 2c, Supplementary Fig. 3b). Gene-expression analysis of $\mathrm{B}^{1} 6^{\mathrm{IDO}}$-isolated TAMs showed reduced expression levels of M1-markers (NOS2, IL12, TNFa, CD86, CD40) and upregulation of the M2-markers STAB1 and TGFB1 (Fig. 2d) compared to B16 $6^{\mathrm{WT}}$. TAMs accumulated over time as tumors progressed while retaining their M2-status (Supplementary Fig. 3c) and IDO overexpression promoted a higher frequency of TGF- $\beta$-expressing DCs $(P=0.02)$ relative to control (Supplementary Fig. 3d), consistent with a tolerogenic phenotype ${ }^{22}$. Immunophenotyping analysis of $\mathrm{B} 16^{\mathrm{IDO}}$ and $\mathrm{B} 16^{\mathrm{TDO}}$ tumors revealed an upregulation of AHR expression in tumor-infiltrating myeloid-cell populations, such as $\mathrm{Ly} 6 \mathrm{C}^{+}$Monocytes $\left(\mathrm{CD} 11 \mathrm{~b}^{+}\right.$ Ly6 $\left.\mathrm{C}^{\text {high }} \mathrm{Ly} \mathrm{G}^{-}\right)$, conventional DCs $\left(\mathrm{CD} 11 \mathrm{~b}^{+} \mathrm{CD} 11 \mathrm{c}^{+}\right)$, polymorphonuclear cells $\left(\mathrm{CD}_{11 \mathrm{~b}}{ }^{+} \mathrm{Ly}_{6 \mathrm{G}}{ }^{+} \mathrm{Ly} 6 \mathrm{C}^{\text {low }}\right)$ and particularly TAMs in comparison to B16 WT tumors (Supplementary Fig. 3e). Gene-expression analysis revealed an upregulated expression of the CYP1A1 and CYP1B1 genes in B16 ${ }^{\mathrm{IDO}}$ TAMs, indicating engagement of the AHR pathway (Fig. 2e). In addition, we found their more potent $\mathrm{T}$ cell inhibitory function than TAMs isolated from $\mathrm{B} 16^{\mathrm{WT}}$, as evidenced by their ability to modulate the expression of activation markers (CD44, PD1) in autologous $\mathrm{CD}^{+} \mathrm{T}$ cells in vitro (Fig. $2 \mathrm{f}$ ).

Previous studies revealed that the presence of immunosuppressive cell types, including MDSCs and M2-like TAMs, plays a critical role in limiting responses to $\mathrm{ICB}^{23,24}$, while their depletion enhances antitumor immunity ${ }^{25,26}$. We therefore investigated the potential interplay between Kyn-AHR pathway and myeloid cell-mediated $\mathrm{T}$ cell inhibition. Tumor progression rate of $\mathrm{B} 16^{\mathrm{IDO}}$ tumors was delayed in AHR deficient mice $\left(\mathrm{AHR}^{\mathrm{KO}}\right)$ (Supplementary Fig. 3f) and depletion of macrophages with aCSF1-R (Supplementary Fig. 3g-h) or clodronate liposomes (Fig. 2g) delayed the progression of IDO-expressing tumors but not wild-type tumors. Tumor progression delay after macrophage depletion was reversed upon $\mathrm{CD}^{+} \mathrm{T}$ cell depletion (Supplementary Fig. 3i), suggesting that TAMs support tumor progression primarily by inhibiting $\mathrm{CD}^{+} \mathrm{T}$ cell immunity. 
To further assess the effects of Kyn-mediated activation of AHR on macrophage effector function, we cultured Kyn-treated bone marrow-derived antigen-presenting cells and observed decreased expression of the antigen-presenting machinery proteins MHC Class I and II, and the co-stimulatory molecule CD86 (Supplementary Fig. 4a). This effect was reversed upon treatment with the AHR inhibitor CH-223191 (Supplementary Fig. 4b). In addition, higher levels of certain inflammatory cytokines, including IL-1 $\beta$, IL- 6 , and VEGF, were observed in the culture media after treatment with Kyn in a dose-dependent manner (Supplementary Fig. 4c). To assess AHR-mediated changes in antigen presentation function by APCs, which could contribute to the impaired immunity in the IDO/TDO-expressing models, we co-cultured carboxyfluorescein succinimidyl ester (CFSE)-labeled pmel1.1 CD8 ${ }^{+} \mathrm{T}$ cells (pmels) with gp100-loaded BM-APCs pre-exposed to increasing concentrations of Kyn. We observed a significant decrease in the proliferation rate (CFSE ${ }^{\text {low }}$ ) and activation state $(\mathrm{CD} 44, \mathrm{GzmB})$ of pmels cultured in the presence of AHR-activated BM-APCs in a dose-dependent manner (Supplementary Fig. 4d). Culture of pmels with $\mathrm{AHR}^{\mathrm{KO}}$ BM-APCs increased their activation profile $\left(\%\right.$ of $\left.\mathrm{CD} 44^{+} \mathrm{CD} 25^{+}\right)$ in comparison to control (Supplementary Fig. 4e) while AHR ${ }^{\mathrm{KO}}$ BM-APCs presented a less immunoregulatory phenotype, with downregulation of PD-L1 and CD206 (Supplementary Fig. 4f). Taken together, our findings demonstrate that Kyn-mediated activation of AHR is capable of impairing the effector function of macrophages and antitumor $\mathrm{T}$ cell immunity.

Kyn-AHR signaling promotes Treg and TAM cooperative effects. Consistent with recent reports ${ }^{2}$ and our previous results (Fig. 1 and Supplementary Fig. 1C), IDO-expressing tumors presented an increased Treg frequency and upregulated expression of FoxP3 in intratumoral CD4 ${ }^{+} \mathrm{T}$ cells from in relation to B16 ${ }^{\mathrm{WT}}$ (Fig. 3a). The Kyn-AHR pathway was previously implicated in epigenetic regulation of FoxP3 $3^{27}$ and in the generation of $\mathrm{Tregs}^{28}$. In our pre-clinical model, Tregs seem to play a major role in mediating $\mathrm{B} 16^{\mathrm{IDO}}$ tumor progression given that the selective depletion of Tregs, using a transgenic mouse strain engineered with a transgene that expresses the diphtheria toxin receptor (DTR) under control of the Foxp3 promoter (Foxp 3 DTR mice), led to regression of $\mathrm{B} 16^{\mathrm{IDO}}$ tumor growth (Fig. 3b). B16 $6^{\mathrm{IDO}}$ Tregs had increased expression of AHR and the AHRtarget AHR repressor gene $(A H R R)(P=0.0003)$, as compared to WT Tregs (Fig. 3c). Tregs isolated from B16 $\mathrm{IDO}$ tumors, implanted in transgenic mice expressing Foxp3-GFP fusion protein (Foxp3 ${ }^{\mathrm{GFP}}$ knock-in mice), were superior in their ability to suppress the proliferation and activation of of autologous $\mathrm{CD} 8^{+} \mathrm{T}$ cells in vitro when compared with $\mathrm{B} 16^{\mathrm{WT}}$-isolated Tregs (Fig. 3d). On the transcriptional level, Tregs isolated from the B16 ${ }^{\mathrm{IDO}}$ tumors exhibited a more prominent expression of the inhibitory factors such as IL-10, VEGF, CTLA-4, as well as upregulation of Ikzf2 (Helios), OX40, and Ki67 (Fig. 3e). Interestingly, bead array-based analysis of culture supernatants from $\mathrm{B} 16^{\mathrm{IDO}}$ Tregs stimulated with anti-CD3/CD28 revealed increased levels of the chemokines MIP-1 alpha, MIP-1 beta, RANTES and of IFN $\gamma$ (Supplementary Fig. 5a).

Specific depletion of Tregs using Foxp $3^{\mathrm{DTR}}$ mice significantly abrogated the myeloid-enriched phenotype found in B16 $\mathrm{IDO}$ tumors, with reduced infiltration of $\mathrm{CD} 11 \mathrm{~b}^{+}$cells and M2-like TAMs after treatment with DT (Fig. 3f). These data suggest that Tregs may promote a protumoral TAM activity, similar to what described in other recent reports ${ }^{29,30}$. Consistently, RNAseq analysis of human cancers revealed a strong positive correlation between the expression of Treg markers (FOXP3, IL2RA) and myeloid cell markers (MRC1, CD14, and ITGAM) across several tumor types (Fig. 3g). In melanoma, the correlation was particularly strong in tumors expressing high levels of IDO (IDOhigh) and non-existent in those expressing low levels of IDO (IDOlow), with similar observations when categorizing tumors by CYP1B1 expression (Fig. 3h, Supplementary Fig 5b). Accordingly, a similar correlation between Tregs $\left(\mathrm{CD} 4{ }^{+} \mathrm{FoxP}^{+}\right)$and $\mathrm{CD} 11 \mathrm{~b}^{+}$ cells was detected in IDO high melanoma cell suspensions as compared to IDOlow samples (Fig. 3h). With that, we hypothesized that an interplay between Tregs and TAMs exist, with the Kyn-AHR signaling playing a role in promoting such immunoregulatory axis. To assess if Tregs could induce changes in the activation state of myeloid cells through the activation of AHR, we made use of Treg-myeloid cell co-culture models. Pretreatment of ex vivo splenic Tregs isolated from Foxp3 $3^{\mathrm{GFP}}$ hosts with Kyn led to expansion of M2-like macrophages $\left(\mathrm{CD}_{206}{ }^{+}\right.$and $\mathrm{PD}-\mathrm{L} 1^{\text {high }}$ ) in co-cultures. The expansion of the M2-like macrophages was reversed with selective AHR inhibitor $(\mathrm{CH}-$ 223191) (Supplementary Fig 5c). Moreover, AHR ${ }^{K O}$ Tregs presented diminished inhibitory function over $\mathrm{T}$ cell activation and the engagement of AHR with increasing concentrations of Kyn in WT Tregs, but not $\mathrm{AHR}^{\mathrm{KO}}$ Tregs, led to superior suppressive function over $\mathrm{T}$ cell priming by BM-APCs (Supplementary Fig $5 \mathrm{~d}-\mathrm{e})$. Taken together, our data suggest that an engagement of the AHR pathway by IDO or TDO-derived Kyn can drive a Treg-macrophage suppressive axis and tumor progression.

AHR blockade reverses IDO/TDO-mediated immunosuppression. Based on these findings, we sought to explore the effects of using selective AHR inhibitors in controlling tumor growth. Since cancer cells can preferentially up-regulate IDO or TDO, and the release of Kyn is downstream of both pathways, we reasoned that pharmacologic AHR inhibition might lead to superior immune effects, compared with selective IDO or TDO inhibition strategies in controlling IDO/TDO-expressing tumor growth by ensuring that both downstream pathways are blocked (Fig. 4a). Treatment with Epacadostat, a selective inhibitor of IDO, led to inhibition of growth of $\mathrm{B} 16^{\mathrm{IDO}}$, but not of $\mathrm{B} 16^{\mathrm{WT}}$ or $\mathrm{B} 16^{\mathrm{TDO}}$ tumors, while $680 \mathrm{C} 91$, a specific TDO inhibitor, led to delayed progression of $\mathrm{B} 16^{\mathrm{TDO}}$, but not of $\mathrm{B} 16^{\mathrm{WT}}$ or $\mathrm{B} 16^{\mathrm{IDO}}$ tumors. In contrast, treatment with an AHR inhibitor (AHRil, CH-223191) led to tumor growth inhibition in both $\mathrm{B} 16^{\mathrm{IDO}}$ or $\mathrm{B} 16^{\mathrm{TDO}}$ models, but not B16 ${ }^{\mathrm{WT}}$ (Fig. 4a). Similar results were observed using clofazimine, a recently described antagonist of the $\mathrm{AHR}^{31}$, and KYN101, an optimized, selective synthetic antagonist of AHR (Supplementary Fig. 6a). KYN-101 shares pharmacological properties with the AHR antagonist selected for clinical development in cancer patients. The clinical candidate is similar in potency, selectivity and in vivo activity to that demonstrated with KYN101 (Supplementary Fig. 7). Gene-expression analysis of the whole tumors after AHRil treatment revealed upregulation of GZMB, CD86, and IFNG in B16 ${ }^{\mathrm{IDO}}$ tumors in comparison to control (Supplementary Fig 6b) which is consistent with a previous report implicating AHR signaling in shaping IFN-mediated responses ${ }^{32}$. We also found that the treatment of $\mathrm{B} 16^{\mathrm{IDO}}$ tumors with AHRil in immunodeficient mice $\left(\mathrm{Rag}^{-/}\right.$) did not completely abolish its therapeutic efficacy, suggesting that a non-T cell TME component is potentially involved in the antitumor effect of AHR inhibitors (Supplementary Fig. 6c).

Immunophenotypic analysis of $\mathrm{B} 16^{\mathrm{IDO}}$ tumors after AHRi1 treatment revealed repolarization of TAMs, as characterized by increased MHCII expression (Fig. 4b), and an increase in antigen-specific $\mathrm{T}$ cell priming. AHRil treatment resulted in an increase in adoptively transferred pmels and their activation profile within tumors and tumor-draining lymph nodes (TDLN) 
a

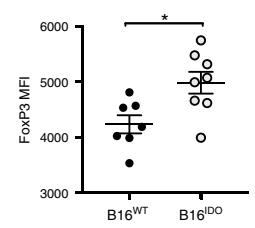

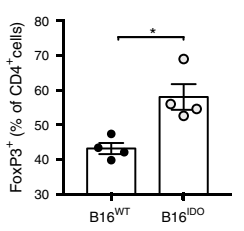
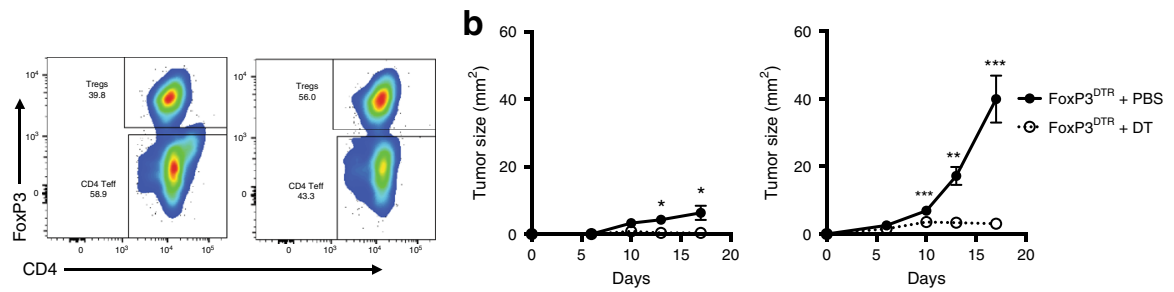

C
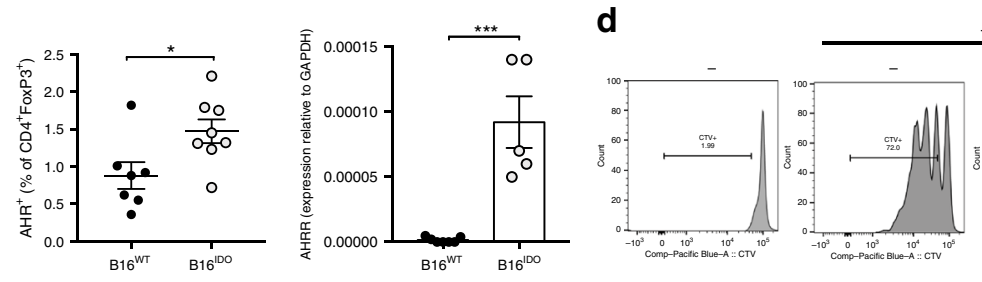

$+\mathrm{aCD} 3$ and irradiated SPL

$\mathbf{e}$
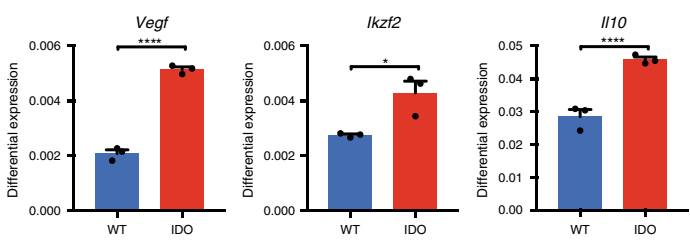

f

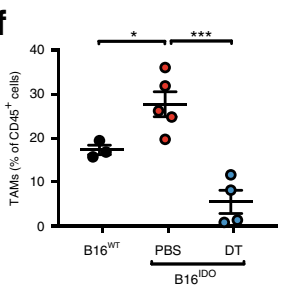

h
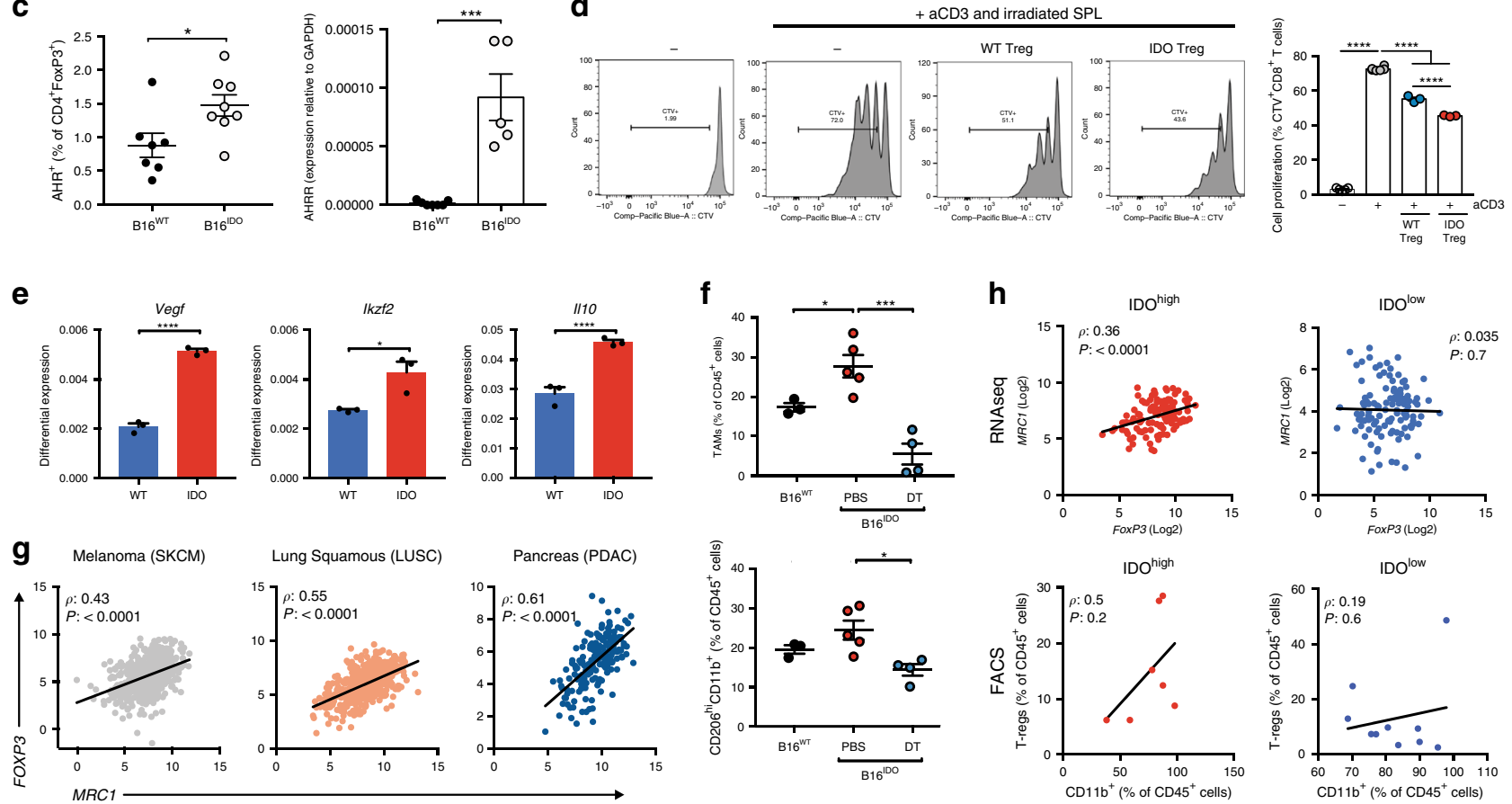

Fig. 3 FoxP3 ${ }^{+}$T cells co-operate with TAMs in promoting immune suppression of IDO + tumors. a Flow cytometric analysis of FoxP3 expression in tumor-infiltrating $\mathrm{CD}^{+}{ }^{+}$populations (left), and of Tregs $\left(\mathrm{CD} 4^{+}\right.$FoxP3 $\left.{ }^{+}\right)$(right) $\left(n=7\right.$ (B16 $\left.{ }^{\mathrm{WT}}\right)$ and $n=8$ (B16 IDO)). Results representative of three independent experiments. b Tumor progression of B16WT (left) and B16IDO (right) tumors implanted in FoxP3DTR mice treated with PBS or DT (1 $\mu$ g/ mouse) ( $n=5$ per group). c FACS analysis of AHR expression (left) ( $n=7$ (B16WT) and $n=8\left(B 16^{I D O}\right)$ ) and mRNA analysis by qRT-PCR analysis of AHRR expression in B16 IDO and B16WT tumor-isolated T-regs (right). d In vitro suppressive activity of tumor-isolated Tregs isolated from B16 ${ }^{\mathrm{IDO}}$ or B16 WT.

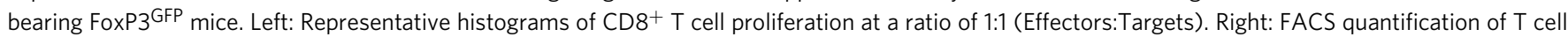
proliferation (CTVlow) $(n=3)$. e mRNA of Treg markers by qRT-PCR analysis in tumor-isolated Tregs from B16WT and B16 IDO tumors ( $n=3$ ). $\mathbf{f}$ FACS

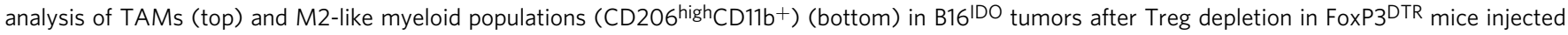
with DT (1 $\mu \mathrm{g} /$ mouse) or PBS (B16WT $n=3$; B16IDO PBS $n=4$, DT $n=5$ mice/group). $\mathbf{g}$ Correlation analysis between myeloid marker (MRC1/CD206) and

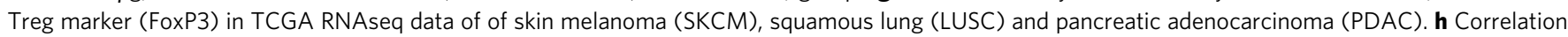
analysis in TCGA RNAseq data of IDO high and IDO low skin melanomas (SKCM) analyzed by Spearman rank correlation. Bottom: correlation analysis between frequency of Tregs (CD4 ${ }^{+}$FoxP3 $^{+}$) and myeloid cells (CD11b ${ }^{+}$) in FACS analyzed IDOhigh and IDOlow melanoma patients' biopsies by Spearman rank correlation. Results in (b-f) are representative of two independent experiments. Data is represented as mean values \pm SEM. Two-tailed unpaired

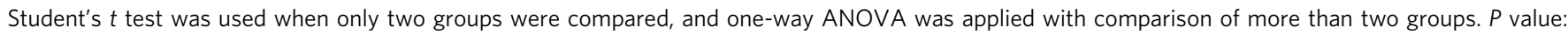
${ }^{\star} P<0.05,{ }^{\star \star} P<0.01,{ }^{\star \star \star} P<0.001,{ }^{\star \star \star \star} P<0.0001$.

of $\mathrm{B} 16^{\mathrm{IDO}}$-bearing mice (Supplementary Fig. 6d). In addition, treatment with AHRil led to decreases in the frequency of intratumoral Tregs (Fig. 4c). Gene-expression analysis of intratumoral Tregs and effector CD4 $+\mathrm{T}$ cells (Teff) revealed a markedly differential gene-expression signature after AHRil treatment, consistent with a dysfunctional and less suppressive state in Tregs and Th-1-like effector phenotype in Teff in both $\mathrm{B} 16^{\mathrm{IDO}}$ and $\mathrm{B} 16^{\mathrm{TDO}}$ models (Supplementary Fig. 8a-b).

We found that intratumoral $\mathrm{CD}^{+} \mathrm{T}$ cells from the AHRil group had increased expression of the proliferation marker Ki67, the transcription factor T-bet (Supplementary Fig. 8c) and were enriched in $\mathrm{PD}-1^{+} \mathrm{Ki} 67^{+}$cells (Fig. 4d), which has been recently linked with a $\mathrm{T}$ cell reinvigoration phenotype $\mathrm{p}^{33}$. In addition, we observed an increased population of $\mathrm{CD}^{+} \mathrm{T}$ cells with a marked increased in a double-positive Eomes ${ }^{+}{ }^{\text {T-bet }}{ }^{+}$cells
(Supplementary Fig. 8d), that was previously described as characteristic of a reversibly exhausted population ${ }^{33}$. Similar immunomodulatory effects of AHRil treatment were observed in the B16 $16^{\text {TDO }}$ model (Supplementary Fig. 9). FACS sorting of intratumoral $\mathrm{CD}^{+} \mathrm{T}$ cells from $\mathrm{B} 16^{\mathrm{IDO}}$-bearing mice after treatment with AHRil revealed increased expression of proinflammatory cytokines (IFN- $\gamma$, IL-10, MIP1a, and MIP1b) after ex vivo stimulation (Supplementary Fig. 8e). Given the immune modulatory effects of AHR inhibition in the TME of IDO/TDOexpressing models, we next evaluated if its combination with immune checkpoint blockade (anti-PD-1) could yield additional antitumor activity. Use of AHRi1 (CH-223191) with anti-PD-1 led to delayed tumor progression and prolonged survival in $\mathrm{B} 16^{\mathrm{IDO}}$ and $\mathrm{B} 16^{\mathrm{TDO}}$ tumors models when compared with single agent therapy (Fig. 4e). On the other hand, addition of AHRil did 
a

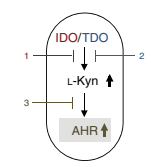

${ }^{B 1} 6^{W T}$
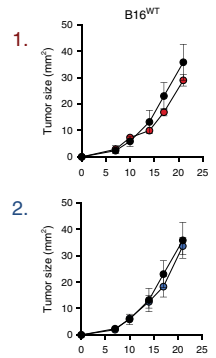

3.

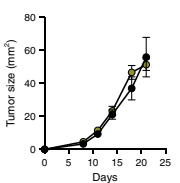

b

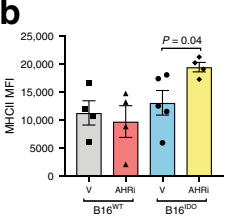

d

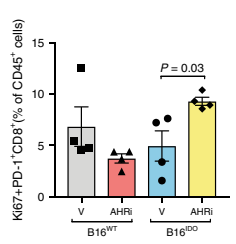

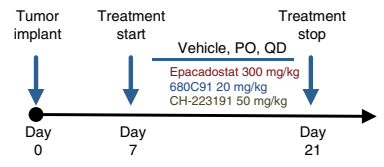
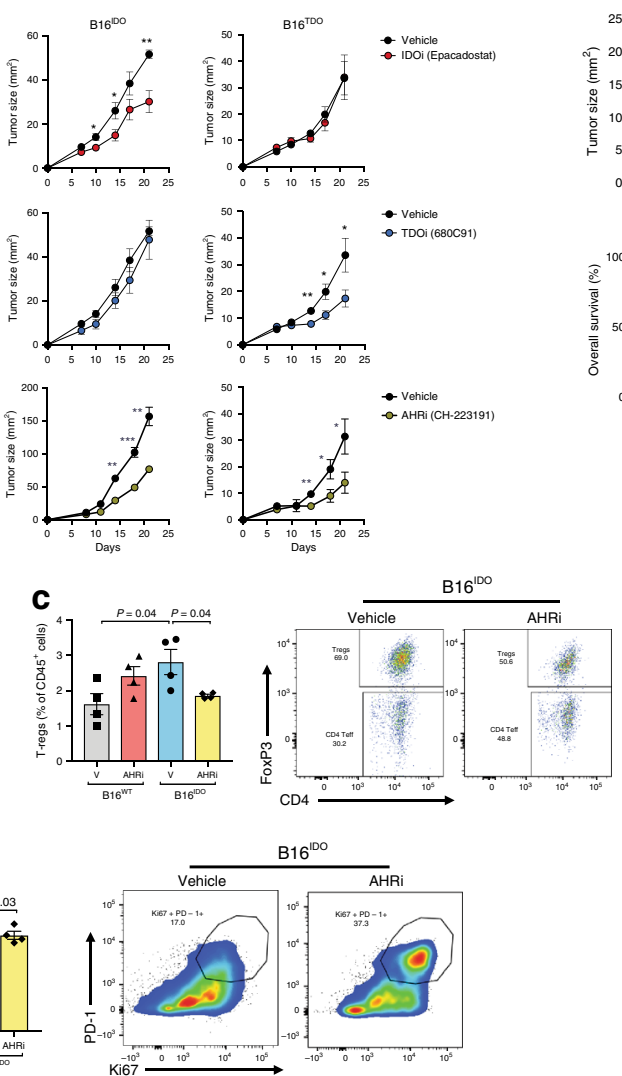

e
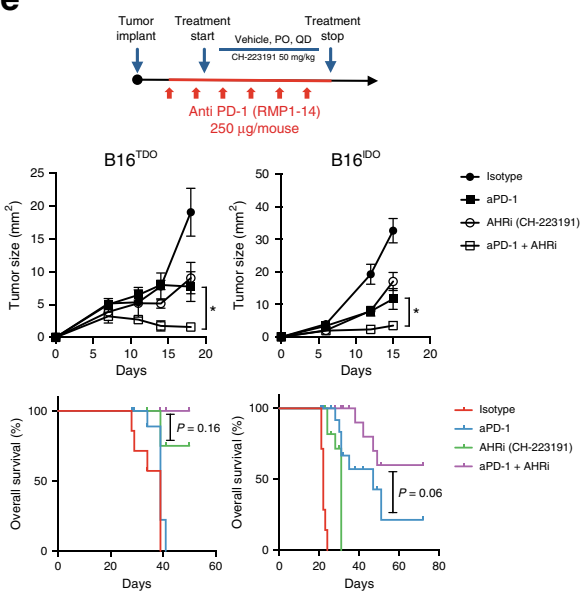

$\mathbf{f}$
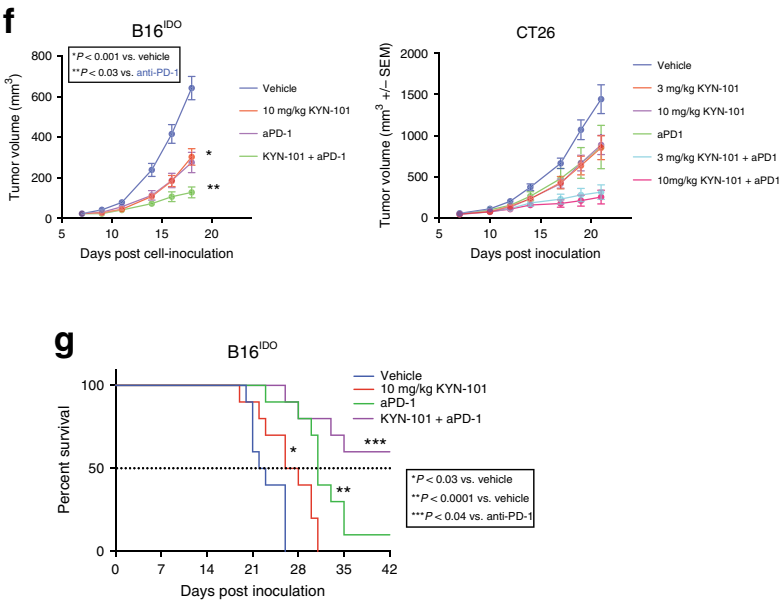

Fig. 4 Selective AHR inhibition reverses IDO/TDO-mediated tumor progression and improves the efficacy of PD-1 blockade. a Top left: scheme figure with experimental approach of targeting multiple steps of Trp-catabolic pathway. Top right: therapy regimen (PO, QD). Bottom: tumor progression of orthotopically injected B16WT, B16IDO and B16 TDO tumors in mice treated with vehicle or inhibitors (Epacadostat/IDO inhibitor 300 mg/kg/d, 680C91/ TDO inhibitor $20 \mathrm{mg} / \mathrm{kg} / \mathrm{d}, \mathrm{CH}-223191 / \mathrm{AHR}$ inhibitor $50 \mathrm{mg} / \mathrm{kg} / \mathrm{d}$ ). ( $n=5$ mice per group for two independent experiments). b FACS analysis of MHCII expression ( $n=4$ per group, $n=5$ B16 ${ }^{\mathrm{IDO}}$ vehicle). c Quantification of frequency and representative plots of intratumor Tregs (CD4+FoxP3+CD25+/\% of

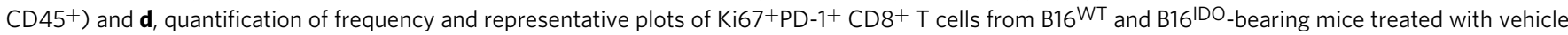
or AHR inhibitor ( $\mathrm{CH}-223191)$ ( $n=4$ per group). e Top: therapy regimen. bottom: mean tumor size and overall survival of B16 ${ }^{\text {IDO }}$ and B16 ${ }^{\text {TDO }}$ tumorbearing mice treated with $\mathrm{AHR}$ inhibitor $(\mathrm{CH}-223191)$, anti-PD-1 alone or in combination with $\mathrm{AHR}$ inhibitor (combo) ( $n=5$ B16 TDO and $n=10$ B16IDO). f Mean tumor size of B16IDO and CT26 and $\mathbf{g}$, overall survival of B16IDO tumor-bearing mice treated with the optimized AHR inhibitor (KYN-101), anti-PD-1 alone or in combination with AHR inhibitor (combo) ( $n=10$ mice per group). Results are representative of two independent experiments. Data represented as mean values \pm SEM. Two-tailed Student's $t$ test was used when only two groups were compared and log-rank (Mantel-Cox) test was used for survival comparison. $P$ value: ${ }^{\star} P<0.05,{ }^{\star \star} P<0.01,{ }^{\star \star \star} P<0.001,{ }^{\star \star \star \star} P<0.0001$.

not improve the response to anti-PD-1 alone in a model lacking IDO/TDO-Kyn pathway activation (B16 ${ }^{\mathrm{WT}}$ ) (Supplementary Fig. 10a). In addition, tumor-free survivors in the combination therapy group were resistant to tumor re-implantation, indicating the development of long lasting adaptive immunity (Supplementary Fig. 10b). Combination of AHRi and anti-PD-1 increased markers of activation in intratumoral CD8 ${ }^{+} \mathrm{T}$ cells $(\mathrm{CD} 44, \mathrm{GrzB}$ and Ki67) and myeloid cells (MHCII, PD-L1) (Supplementary Fig. 10c-d). Interestingly, an optimal efficacy of AHRi and PD-1 blockade was dependent on the presence of myeloid cells (monocytes/macrophages), as their depletion using aCSF1R or using CCR2 ${ }^{\mathrm{KO}}$ mice attenuated the efficacy of the combination AHRi1+ anti-PD-1 therapy (Supplementary Fig. 10e).

Furthermore, we found that the combination of KYN-101 (AHRi2) and anti-PD-1 led to improved tumor growth delay and extended survival in $\mathrm{B} 16^{\mathrm{IDO}}$ (Fig. 4f, g). Similar oberservation was made in the CT26 colorectal cancer model expressing endogenous high levels of IDO (Fig. 4f). Thus, these data indicate that the reversion of a regulatory phenotype in immune cells can be achieved by selective pharmacologic targeting of the AHR to promote more effective antitumor immunity and enhance the effects of PD-1 blockade.

\section{Discussion}

Upregulation of IDO, together with other inhibitory checkpoint molecules such as PD-L1, LAG-3, and VISTA, are welldocumented mechanisms of acquired resistance to immunotherapy ${ }^{24}$. A high frequency of IDO and TDO expression (32 and $35 \%$ respectively ${ }^{34}$ ) is described in multiple cancer types, such as melanoma, lung cancer, pancreatic cancer and renal cell carcinoma $^{35}$. Given the mouting evidence that high IDO expression can lead to inhibition of the effector function of $\mathrm{T}$ cells, either directly or indirectly through non- $\mathrm{T}$ cell components ${ }^{36,37}$, strategies to effectively target this metabolic 
pathway can reverse tumor immune resistance mechanisms. Interestingly, increases in the IDO/TDO-product Kyn in the serum of patients receiving anti-PD-1 were recently reported as an adaptive resistance mechanism associated with worse overall survival ${ }^{13,38}$. Similarly, we here found that upregulation of Kyndegrading enzymes correlates with good response to anti-PD-1 therapy (Fig. 1a). Therefore, identification of mechanisms that control the response to oncometabolites such as Kyn in the TME is likely to guide new therapeutic approaches. In this study, we provide evidence that the IDO/TDO-downstream AHR activation represents a common feature of cancers overexpressing IDO or TDO, which dictates a $\mathrm{T}$ cell suppressive TME through the establishment of a Treg-macrophage suppressive axis.

As reported previously, resistance to ICB therapy is commonly associated with high Treg/Teff ratio $^{39,40}$ and strong immunosuppressive signatures conferred by macrophages, including angiogenesis and T cell suppressing soluble factors ${ }^{41}$. AHR is known to play an important role in modulation of immune responses and to be expressed by several cell types ${ }^{42}$. Our profiling of the TME indicates that Tregs and TAMs retain an immunosuppressive pro-tumoral function through the engagement of the AHR pathway in the presence of high IDO or TDO expression. A recent paper using a glioblastoma model showed an AHR-driven upregulation of the ectonucleotidase CD39 in TAMs which promoted T cell dysfunction ${ }^{21}$. In our study, changes detected in Tregs and TAMs were both quantitative and qualitative, as described in Figs. 2 and 3, and can account to the highly dysfunctional effector phenotype of $\mathrm{CD}^{+} \mathrm{T}$ cell in these IDO/TDO-expressing models. Although a causal role for low Trp levels in $\mathrm{T}$ cell inhibition cannot be fully discarded, the macrophage and Treg-dependent tumor progression detected in these models and its reversion upon AHRblockade suggest that indirect TME-related mechanisms of T cell suppression drive IDO/TDO-mediated cancer progression through the AHR pathway.

In addition, our findings that an induction of AHR signaling in Tregs can modulate M2-like TAMs activity are interesting and reveal an undescribed role of AHR signaling in mediating immune resistance. Although the effects of AHR signaling can take place on multiple immune cells ${ }^{43}$, AHR activation in Tregs may be contributing to the establishment of a myeloid-enriched immunosuppressive TME. Other similar Treg-TAM interactions were shown in the context of cancer and other inflammatory diseases in recent publications, with Tregs promoting macrophage efferocytosis ${ }^{30}$ and accumulation of M2-like TAMs in the TME indirectly by limiting the secretion of the M1-inducer signal IFN $\gamma$ in $\mathrm{CD}^{+} \mathrm{T}$ cells ${ }^{29}$. Although we identified AHR signaling as a key molecular mechanism controlling this Treg-macrophage interplay, further studies will be necessary to better elucidate this regulatory pathway.

In a previous report, activation of the AHR by tobacco smoke was able to induce PD-L1 expression and immune-escape in a lung cancer model ${ }^{44}$ and AHR activity predicted response to PDL1 blockade. Here, we identified a common metabolic mechanism that participates in the control of Tregs, TAMs and T cell immunity in the context of IDO or TDO expression. Furthermore, we show that the efficient and selective targeting of the AHR pathway in tumors with an active Trp catabolic pathway overcomes immunosuppressive features in the TME and can sensitize otherwise immune resistant tumors to clinically approved PD-1 blockade. Our results provide a rationale for assessing active metabolic pathways in tumors, such as by monitoring the circulating levels of Kyn and Kyn/Trp ratio as recently reported $^{38}$ in a precision-medicine type of approach, for the design of combination strategies using AHR inhibitors in future clinical trials.

\section{Methods}

Cell lines. The murine cancer cell lines for melanoma (B16F10, referred to as B16), and colon cancer (CT26) were obtained from ATCC. Cells were maintained in RPMI medium supplemented with $10 \%$ fetal calf serum (FCS) and penicillin with streptomycin (complete RPMI media). $\mathrm{B} 16^{\mathrm{IDO}}$ and $\mathrm{B} 16^{\mathrm{TDO}}$ cells were generated by transduction of B16F10 with GFP plus the IDO gene or TDO gene in the pCMV6AC-GFP vector (Origene). B16F10 transduced with GFP alone were used as control cells (B16-WT). Clonal transformants were selected using $1 \mathrm{mg} / \mathrm{ml}$ G418 (Geneticin). Cell lines were routinely screened to avoid mycoplasma contamination and maintained in a humidified chamber with $5 \% \mathrm{CO}_{2}$ at $37^{\circ} \mathrm{C}$ for up to 1 week after thawing before injection in mice.

Quantitative RT-PCR or mRNA expression profiling. Cells were lysed in TRIzol LS reagents (Life technologies) and frozen at $-80^{\circ} \mathrm{C}$ for future use. Process of total RNA extraction was performed according to the manufacturer's instructions. Reverse transcription to cDNA was performed with $1 \mu \mathrm{g}$ using a High Capacity cDNA Archive Kit (Life technologies). cDNA samples were pre-amplified using the PreAmp master mix (Fluidigm) and the mixtures of all the Taqman assays (lifetechnologies) to prepare preamplified cDNA by PCR (18 cycles), following the manufacturer's instruction. Pre-amplified samples were loaded in a 48.48 dynamic array integrated fluidic circuit (IFC; Fluidigm) using an MX IFC controller (Fluidigm). mRNA expression data were processed by BioMark HD System and data analyzed using the real-time PCR analysis software (Fluidigm). mRNA levels were normalized to Gapdh and data were analyzed by applying the $2^{\wedge}(-\mathrm{dCt})$ calculation method. A list of primers can be found in Supplementary Table 1.

Cytokine analysis. Tumors were excised, snap-frozen in dry ice, and pulverized using a tissue grinder. Tumor protein homogenates were prepared in PBS containing $2 \times$ Halt Protease and Phosphatase Inhibitor Cocktail (Fisher Scientific). Total protein concentration was normalized to $2 \mathrm{mg} / \mathrm{ml}$. For cell supernatants, they were collected from cell culture, centrifuged at $4 \mathrm{C}$ for $15 \mathrm{~min}$ at high speed to remove cells and frozen in $-80 \mathrm{C}$ freezer until the day of the experiment. Cytokines were quantified using the MILLIPLEX MAP Mouse Cytokine/Chemokine Magnetic Bead 32 Plex Panel according to the manufacturer's instructions (Millipore, Billerica, Massachusetts).

Mice. C57BL/6 and Balb/c mice (6-8-weeks old), $\mathrm{Rag}^{-1-}$ mice and CCR2 ${ }^{-1-}$ on C57BL/6 background were purchased from Jackson Laboratory. Constitutive AHR knockout mice were obtained from Taconic. Pmel-1 TCR transgenic mice have been reported ${ }^{45,46}$ and were provided by N. Restifo (National Cancer Institute (NIH), Bethesda, MD). Foxp3 ${ }^{\text {GFP }}$ knock-in mice were a gift from Dr. A. Rudensky (Memorial Sloan Kettering Cancer Center (MSKCC), New York, NY). Foxp3 DTR mice were generated in the laboratory of Dr. G. J. Hammerling (German Cancer Research Center (DKFZ), Heidelberg, Germany), and previously described ${ }^{47}$. All mice were maintained in microisolator cages and treated in accordance with the NIH and American Association of Laboratory Animal Care regulations. All mouse procedures and experiments for this study were approved by the MSKCC Institutional Animal Care and Use Committee.

Tumor challenge and treatment experiments. On day 0 of the experiments, tumor cells were injected intradermally (i.d.) in the right flank. $2 \times 10^{5} \mathrm{~B}^{\mathrm{WTT}}, \mathrm{B} 16^{\mathrm{IDO}}$ or $\mathrm{B}^{\mathrm{TDO}}$ were injected into C57BL/6J mice. For studies in immune compromised mice, the study was done in C57Bl.6 Rag1 ${ }^{-/}$mice. Mice were obtained from Jackson Labs and Charles River Labs. Treatments were given as single agents or in combinations with the following regimen for each drug. The AHR inhibitor drug $\mathrm{CH}-223191$ was dissolved at $0.5 \%$ methyl cellulose (MC) and $0.5 \%$ Tween 80 in water and administered by oral gavage once a day at $50 \mathrm{mg} / \mathrm{mg}$. Treatment was initiated on day 7 ending on day 21 post tumor implantation. Control groups received vehicle $(0.5 \% \mathrm{MC}, 0.5 \%$ Tween 80) without the active product. For Clofazimine, treatment was initiated on day 7 ending on day 21 post tumor implantation. Clofazimine was prepared in a solution of $5 \%$ DMSO in PBS and mice were treated with daily i.p. injections at $10 \mathrm{mg} / \mathrm{kg}$ as described $^{31}$. Epacadostat was dissolved in a $0.5 \%$ MC vehicle solution and $680 \mathrm{C} 91$ in a $5 \%$ DMSO $+5 \%$ Solutol solution in water and administered daily by oral gavage at $300 \mathrm{mg} / \mathrm{kg}$ and $20 \mathrm{mg} / \mathrm{kg}$, respectively. Anti-PD-1 antibody $(250 \mu \mathrm{g}$ per mouse, clone RPM1-14, Bio X cell) was injected intraperitoneally (i.p.) every 3 days, starting from day 3 after tumor implantation. Treatment with anti-CSF1R (BioXCell) at $300 \mu \mathrm{g} /$ mouse and anti-CD8 (2.43) at $250 \mu \mathrm{g} /$ mouse was started on day 3 post tumor challenge for every 3 days. Tumors were measured every second or third day with a calliper, and the tumor size was calculated based on an ellipsoid formula. Mice that had no visible and palpable tumors that could be measured on consecutive measurement days were considered complete regressions. Animals were euthanized for signs of distress or when the total tumor volume reached $2500 \mathrm{~mm}^{3}$. For the rechallenge study: mice with complete responses in the anti-PD-1 and CH-223191 combination group were re-challenged with $1 \times 10^{5}$ B16-F10 tumor cells (on day 80 of the study since original tumor implant).

AHR antagonism with KYN-101. A novel AHR antagonist, KYN-101 that is potent and selective inhibitor has been developed by Ikena Oncology, formerly known as Kyn Therapeutics. KYN-101 has an $\mathrm{IC}_{50}$ of $22 \mathrm{nM}$ in the human HepG2 
DRE-luciferase reporter assay and $23 \mathrm{nM}$ in the murine Hepal Cyp-luc assay. For efficacy studies with B16-F10 melanoma model, $\mathrm{C} 57 \mathrm{Bl} / 6$ female mice were inoculated intradermally in the hind flank with B16 ${ }^{\mathrm{IDO}}$ tumor cells at $2 \times 10^{5}$ cells/ mouse in a $50 \mu \mathrm{L}$ injection volume. Seven days after cell inoculation, animals were randomized into 4 groups. Animals were dosed with vehicle, KYN-101, anti-PD1, or a combination of anti-PD1 and KYN-101. KYN-101 $(10 \mathrm{mg} / \mathrm{kg})$ and vehicle control $(0.5 \% \mathrm{MC})$ were administered PO daily (QD) for a total of 12 doses. AntiPD1 (250 $\mu \mathrm{g} /$ mouse) and Rat IgG control (in 1X PBS) were administered IP Q3D for a total of 4 doses. Tumor and body weight measurements were taken three times per week. Tumor volumes were calculated, percent inhibition of tumor growth and survival with KYN-101 as a single agent or in combination with antiPD1 compared to vehicle control were determined. For the CT26 colorectal cancer model, Balb/C female mice were inoculated subcutaneously in the hind flank with CT26 tumor cells at $5 \times 10^{5}$ cells/mouse in a $100 \mu \mathrm{L}$ injection volume. Four days after cell inoculation, animals were randomized into 4 groups based on body weight. Animals were dosed with vehicle, KYN-101, anti-PD1, or a combination of anti-PD1 and KYN-101. KYN-101 (3 or $10 \mathrm{mg} / \mathrm{kg}$ ) and vehicle control $(0.5 \% \mathrm{MC})$ were administered PO daily (QD). Anti-PD1 $(10 \mathrm{mg} / \mathrm{kg}$ ) and Rat IgG control (in 1X PBS) were administered IP BIW for a total of 5 doses. Tumor and body weight measurements were taken three times per week. Tumor volumes were calculated and percent inhibition of tumor growth with KYN-101 as a single agent or in combination with anti-PD1 compared to vehicle control were determined.

Kyn measurement. The biological activity of IDO and TDO was evaluated by measuring the level of L-kynurenine by ELISA according to the manufacturer's instructions (LDN Labor Diagnostika Nord and Immundiagnostik). The conditioned medium related to the same number of cells was centrifuged at $12,000 \times g$ for 5 min to remove debris. Samples were read with a SpectraMax 340PC (Molecular Devices) at $450 \mathrm{~nm}$. Samples were read with a SpectraMax 340PC (Molecular Devices) at $450 \mathrm{~nm}$.

Purification of Tregs. Foxp $3^{+}$Tregs were purified from tumors of Foxp3GFP mice. Tumor single-cell suspensions were generated as described previously. CD4+ $\mathrm{T}$ cells were enriched by Ficoll gradient (Sigma-Aldrich), before sorting for GFP expression on a FACSAriaTM II Cell Sorter (BD Biosciences). Dead cells and doublets were excluded on the basis of forward and side scatter and Fixable Viability Dye eFluor 506. Purity of flow-sorted populations was above $90 \%$.

Cell depletions in mice. Foxp $3^{\text {DTR }}$ mice were injected i.p. on days 7-8 and 14-15 after tumor implantation with DT ( $1 \mu \mathrm{g} / \mathrm{mouse}$, Sigma-Aldrich). Clodronate liposomes were injected i.p. twice a week starting on day 3 after tumor implantation ${ }^{48}$.

Isolation of tumor-infiltrating cells and lymphoid tissue cells. Tumor samples were minced with scissors before incubation with $1.67 \mathrm{U} \mathrm{ml}^{-1}$ Liberase (Roche) and $0.2 \mathrm{mg} \mathrm{ml}^{-1}$ DNase (Roche) in RPMI for $30 \mathrm{~min}$ at $37^{\circ} \mathrm{C}$. Samples were then processed by repeated pipetting and filtered through a $100-\mu \mathrm{m}$ nylon filter (BD Biosciences) in RPMI to generate single-cell suspensions, which were subsequently washed with complete RPMI and purified on a Ficoll gradient to eliminate dead cells. Single-cell suspensions from spleens were obtained by grinding spleens through $40-\mu \mathrm{m}$ filters. When required, samples were treated with red blood cell (RBC) lysis buffer (ACK Lysing Buffer, Lonza) and further washed and resuspended in FACS buffer (PBS/0.5\% albumin) before incubation with antibodies.

\section{Generation of bone-marrow derived myeloid cells and priming assay. Isolation} and differentiation of bone marrow-derived antigen-presenting cells (BM-APCs) was performed as previously described ${ }^{49}$. In brief, bone marrow was obtained from mice femurs. After RBC lysis, the cells were plated in six-well plates with a density of $1 \times$ $10^{6} / \mathrm{ml}$ in complete RPMI 1640 media supplemented with $25 \mathrm{ng} / \mathrm{ml} \mathrm{GM-CSF}$ and treated with Kyn at $100 \mu \mathrm{M}$ or DMSO. On days 3 and 6, nonadherent cells and $75 \%$ of culture media were exchanged for fresh media with or without Kyn treatment. A total of $80 \%$ of the cell population stained positive for $\mathrm{CD} 11 \mathrm{c}$ and $\mathrm{F} 4 / 80$ by flow cytometry. For the T cell priming assay, on day $6 \mathrm{BM}$ cells were cultured an additional day to maturity by exchanging $75 \%$ of the media, with the addition of $50 \mathrm{ng} / \mathrm{ml}$ LPS $^{28}$. On day 7, antigen-presenting cells were pulsed with gp100 peptide and co-cultured for 48 hours with freshly isolated melanossomal antigen-specific $\mathrm{CD} 8^{+} \mathrm{T}$ cells (pmel) in a ratio of 10:1 $(1 \times 105 / 0.1 \times 105)$ in a round-bottom 96-well plate (Corning). Pmel CD8 $+\mathrm{T}$ cells were isolated from the spleen of pmel mice by CD8 positive selection through MACS beads (Miltenyi Biotec), labeled with the proliferative tracker CFSE.

Three-dimensional killing assay. The 3D collagen-fibrin gel-based killing assay has been previously described in depth ${ }^{19}$. For the isolation of intratumoral CD8 ${ }^{+} \mathrm{T}$ cells, $\mathrm{B} 16^{\mathrm{WT}}$ and $\mathrm{B} 16^{\mathrm{IDO}}$ tumors were excised on day 15 and dissected into single-cell suspensions, as described above. Tumor-isolated $\mathrm{CD}^{+} \mathrm{T}$ cells were enriched by Ficoll gradient (Sigma-Aldrich) before sorting as $\left(\mathrm{CD}^{2} 5^{+} \mathrm{TCR} \beta^{+} \mathrm{CD} 8^{+}\right)$on a FACSAriaTM II Cell Sorter (BD Biosciences). In brief, $0.1 \times 10^{5}$ viable B16-F10 target cells were coembedded into collagen-fibrin gels with $1 \times 10^{5}$ FACS-sorted effector $\mathrm{CD} 8^{+} \mathrm{T}$ cells in a 10:1 ratio (effector:target). $\mathrm{CD}^{+} \mathrm{T}$ cells isolated from the spleen of Pmel-1/gp100specific TCR transgenic mice were used as a positive control. After $48 \mathrm{~h}$ of co-culture, collagen-fibrin gels were dissolved and target cells were diluted and plated into 6-well plates for a colony formation assay. After 7 days, cells were fixed using $3.7 \%$ formaldehyde and stained with $2 \%$ methylene blue before colony counting.

T cell suppression assay. For the suppression assays with tumor-isolated Tregs, $\mathrm{B} 16^{\mathrm{WT}}$ and $\mathrm{B} 16^{\mathrm{IDO}}$ tumors were grown in Foxp $3^{\mathrm{GFP}}$ mice and Tregs $\left(\mathrm{CD} 45^{+} \mathrm{TCR} \beta\right.$ ${ }^{+} \mathrm{CD} 4{ }^{+} \mathrm{GFP}^{+}$) sorted at day 15 after tumor implantation. Tregs were incubated with CellTrace Violet (CTV, Invitrogen)-labeled target $\mathrm{CD}^{+}{ }^{+} \mathrm{T}$ cells immunomagnetically purified (CD8 Microbeads, Miltenyi Biotec) from spleens of CD45.1+ C57BL/ $6 \mathrm{~J}$ congenic mice. Cultures were stimulated for $48-72 \mathrm{~h}$ with $0.5 \mu \mathrm{g} / \mathrm{ml}$ soluble $\mathrm{aCD} 3$ $\mathrm{Ab}$ and irradiated splenocytes before analyses of target $\mathrm{CD} 8^{+} \mathrm{T}$-cell CTV dilution (proliferation) and CD44 upregulation (activation) by flow cytometry. For the suppression assays with TAMs, B16 ${ }^{\mathrm{WT}}$ and $\mathrm{B} 16^{\mathrm{IDO}}$ tumors were grown in C57BL/ 6J mice. At day 15 after tumor implantation, tumor-isolated macrophages $\left(\mathrm{CD}_{4}{ }^{+}\right.$ $\mathrm{CD}_{11 b^{+}} \mathrm{F} 4 / 80^{\mathrm{hi}} \mathrm{Ly}_{6 \mathrm{G}}$ ) were sorted at over $90 \%$ purity. $\mathrm{CD} 8^{+}$cells isolated from the spleen of naïve mice were purified using anti-CD8 (Ly-2) microbeads (Miltenyi Biotech) according to the manufacturer's protocol. $\mathrm{CD}^{+} \mathrm{T}$ cells were then plated in complete RPMI media supplemented with $0.05 \mathrm{M} \beta$-mercaptoethanol onto round bottom 96 -well plates $\left(1 \times 10^{3}\right.$ cells per well $)$ and were suboptimally stimulated with aCD3/aCD28 microbeads (Dynabeads Human T-Expander CD3/CD28, ThermoFisher). $\mathrm{CD} 8^{+} \mathrm{T}$ cells were plated with FACS-sorted TAMs at a ratio of 2:1 (E:T) for $48 \mathrm{~h}$ before analysis of PD-1 and CD44 upregulation (activation) by flow cytometry.

Isolation of Pmel lymphocytes and adoptive transfer. Spleens and lymph nodes from pmel-1 TCR transgenic mice were isolated and grinded through $100 \mu \mathrm{M}$ filters. After RBC lysis, CD8 + T cells were purified by positive selection using Miltenyi magnetic beads, The cells were labeled with CFSE and injected into recipient animals via tail vein as described in the figure scheme (Fig. 7d). The frequency and activation of pmel cells were measured 7 days after adoptive transfer of $1 \times 10^{6}$ cells using Thy1.1 antibody.

Co-culture Treg-macrophages. Tregs were isolated from spleens of Foxp3 ${ }^{\mathrm{GFP}}$ mice as described above. Cells were maintained with $2000 \mathrm{IU} / \mathrm{mL}$ of IL-2 (Peprotech) and stimulated with plate-bound $\alpha \mathrm{CD} 3(1 \mu \mathrm{g} / \mathrm{mL})$. Treatment was performed with Kyn $(100 \mu \mathrm{M})$ and/or CH-223191 $(10 \mu \mathrm{M})$ for $24 \mathrm{~h}$ prior to the start of co-culture with BMDMs, prepared as described above. Co-culture of pre-treated Tregs with BMDMs was performed for $24 \mathrm{~h}$ at a 1:1 ratio $\left(1 \times 10^{5} \times 1 \times 10^{5}\right.$ cells). For the T cell priming assay, Tregs were isolated as $\mathrm{CD} 4{ }^{+} \mathrm{CD} 25^{+}$using MACS magnetic beads (Miltenyi) from the spleens and lymph nodes of female C57BL/6 mice or $\mathrm{AHR}^{\mathrm{KO}}$ mice at 8-10 weeks of age. Frequency of FoxP3 + cells was over $85 \%$ post purification. BM-APCs on day 7 of GM-CSF maturation (as described above) were cultured in a 96-well round bottom plate coated with anti-CD3 $(0.5 \mu \mathrm{g} / \mathrm{mL})$ with $\mathrm{CD}^{+} \mathrm{T}$ cells on a 10:1 ratio (effector:target). WT or $\mathrm{AHR}^{\mathrm{KO}}$ Tregs were plated on a 2:1 ratio with $\mathrm{CD}^{+} \mathrm{T}$ cells and treatments with Kyn $(50 \mu \mathrm{M})$ and/or CH-223191 $(5 \mu \mathrm{M})$ were added. Cells were analyzed $48 \mathrm{~h}$ later by flow cytometry.

Flow cytometry and morphology analysis. Single cell suspensions obtained from mouse tumors and spleens were pre-incubated $\left(15 \mathrm{~min}, 4^{\circ} \mathrm{C}\right)$ with anti-CD16/32 monoclonal antibody (Fc block, clone $2.4 \mathrm{G}, \mathrm{BD}$ Biosciences) to block nonspecific binding and then stained $\left(30 \mathrm{~min}, 4^{\circ} \mathrm{C}\right)$ with appropriate dilutions of various combinations of the following fluorochrome-conjugated antibodies (clone, dilution, supplier, catalog number): anti-CD206-PE (clone C068C2, 1:200, BioLegend, 141702), anti-MHC Class II (I-A/I-E)-eFluor 450 (clone M5/114.15.2, 1:200, eBioscience, 48-5321-82), anti-MHC Class II-APC-Cy7 (clone M5/114.15.2, 1:200, BioLegend, 107602), anti-CD44-Alexa 700 (clone IM7, 1:200, BioLegend, 103026), anti-PD-1-APC (clone RMP1-30, 1:200, eBioscience,17-9981-82), anti-CD11b-PETR (clone M1/70.15, 1:200, Invitrogen, 50-113-7581), anti-F4/80-BV650 (clone BM8, 1:200, BioLegend, 123149), anti-CD45-AF-700 (clone 30-F11, 1:200, eBioscience, 56-0451-82), anti-CD25-APC-Cy7(clone PC61, 1:200, BD Pharmingen, 557658), anti-CD11c-PE-Cy7 (HL3, 1:200, BD Pharmingen, 550283), anti-PDL1-PE-Cy7 (clone 10F.9G2, 1:200, BioLegend, 124308), anti-CD86-APC (clone GL1, 1:200, BD Pharmingen, 558703), anti-MHC I (H-2Kd)-FITC (clone KH95, 1:200, BD Pharmingen, 553573), anti-TCR $\beta$-APC (clone H57-597, 1:200, eBioscience, 17-5961-82), anti-CD4-BV650 (clone RM4-5, 1:200, BD Horizon, 563747), anti-CD8-PE-TR (clone 5H10, 1:200, Invitrogen, MCD0817), anti-Ly6CPE-Cy7 (clone AL-21, 1:200, BD Pharmingen, 560593), anti-Thy1.1-PE (clone OX7, 1:200, BioLegend, 202524), anti-Ly6G-PercP-Cy5.5 (clone 1A8, 1:200, BD Pharmingen, 560602). For intracellular stain, cells were further permeabilized using a FoxP3 Fixation and Permeabilization Kit (eBioscience) and stained with the antibodies: anti-AHR-PE (clone 4MEJJ, 1:100, eBioscience, 12-5925-82), antiFoxP3-eFluor 450 (clone FJK-16s, 1:200, eBioscience, 48-5773-82), anti-Ki67-FITC (clone SolA15, 1:800, eBioscience, 11-5698-82), anti-T-bet-PE-Cy7 (clone eBio4B10 (4B10, 4-B10), 1:200, eBioscience, 25-5825-82), anti-Granzyme BPECF594 (clone GB11, 1:50, BD Bioscience, 562462), anti-TGF $\beta$-APC (clone 1D11, 1:200, R\&D Systems, MAB1835-100), anti-Eomes-PE (clone Dan11mag, 1:200, eBioscience, 12-4875-82).Stained cells were acquired on a LSRII Flow Cytometer using BD FACSDiva software (BD Biosciences) and the data were processed using FlowJo software (Treestar). Dead cells and doublets were excluded on the basis of forward and side scatter and Fixable Viability Dye eFluor 506. 
Patient material. Patient samples were collected on a tissue-collection protocol approved by the institutional review board of the Memorial Sloan Kettering Cancer Center (IRB \#06-107). Banked tissue samples were retrieved as part of the melanoma disease management biospecimen protocols (IRB\# 00-144 and 19-101). We have obtained informed consent from all participants of this study for sample collection. Specimens from metastatic melanoma patients were processed within a period of 3 hours. Single cell suspensions from tumor samples were obtained by mincing the specimens into small pieces $\left(1 \mathrm{~mm}^{3}\right)$ and further dissociated using enzymatic digestion for $1 \mathrm{~h}$ at $37^{\circ} \mathrm{C}$, with constant shaking in a gentleMACS ${ }^{\mathrm{m}}$ Octo Dissociator. Trypan Blue dye exclusion was used to determine cell viability.

TIL samples were washed and stained with appropriate dilutions of various combinations of antibodies and intracellular IDO staining was performed as described $^{2}$. Melanoma cell suspensions were plated at $0.5 \mathrm{e} 06$ cells $/ \mathrm{mL}$ in 96 -well round bottom plates with treatment with KYN-101 at the concentrations indicated. Cells were acquired $24 \mathrm{~h}$ after for flow cytometry analysis and gene-expression analysis as described above.

Bioinformatic analysis of clinical samples. To determine the expression of kynurenine catabolism enzymes (KMO, KYNU and HAAO), we downloaded the expression data from Riaz et al. ${ }^{15}$. We first calculated the $\log 2$ of each value, and then scaled the $\log 2$ expression values gene-wise to generate $\log 2$ and Z-scaled $\log 2$ values ("Log2" and "Z-Score" in figures, respectively). To determine the average expression of kynurenine degradation enzymes (KD Score), we calculated the mean $Z$-scaled $\log 2$ value of the three enzymes described above for each sample. Shown in the Fig. 1b are the "On Therapy" samples. For gene-expression analysis of AHRrelated genes across immune subtypes in Fig. 1e, the normalized RNAseq expression matrices for the TCGA generated by the Pan Cancer Atlas initiative were downloaded [ref: https://gdc.cancer.gov/about-data/publications/pancanatlas] and the PanImmune Feature Matrix of Immune Characteristics generated were downloaded ${ }^{16}$ that identified and characterized six immune subtypes spanning the tumor types in the TCGA. The log-counts and metadata were subsequently processed in $\mathrm{R}$ and boxplots were made showing ranges of expression across the 6 immune subtypes for select genes using package ggplot. The Wilcoxon signed-rank test was subsequently used to assess any statistical significance in gene expression between immune subtypes. For the correlation analysis, mRNA expression levels were collected from cBioportal (http://www.cbioportal.org/) for skin cutaneous melanoma SKCM (TCGA, Provisional) $(n=472)$, lung squamous cell carcinoma LUSC (TCGA, Provisional) $(n=501)$, pancreas adenocarcinoma PADC (TCGA, Provisional) $(n=179)$ and DLBC (TCGA, $n=48)$ in April 2018. Spearman's rankorder correlation were performed. For the IDO $^{\text {high }}$ and IDO ${ }^{\text {low }}$ analysis, samples were divided into quartiles based on IDO1 expression and Spearman's rank correlation analysis between $M R C 1$ and FOXP3 was performed in the lowest and the highest IDO1 quartile group in $\mathrm{R}$ with the cor.test function. For the survival analysis, patient survival data along with corresponding CYP1B1 mRNA Expression Zscores were downloaded from cBioportal $^{50,51}$ from the provisional Kidney Renal Clear Cell Carcinoma ${ }^{52}$ and Bladder Urothelial Carcinoma ${ }^{53}$ datasets. Patients from each dataset representing the low and high CYP1B1 expression represent the bottom and top quartile, respectively, using the quantile function in R. Survival plots and log-rank statistical test were generated using survfit and ggsurvplot functions from the survival and survminer packages in $\mathrm{R}$.

Statistics and reproducibility. Where indicated, data were analyzed for statistical significance using Prism (GraphPad software, version 7.0) and reported as $P$ values. Data were analyzed with a two-tailed Student's t test when comparing means of two independent groups and one-way ANOVA when comparing more than two groups, as specified. $P<0.05$ was considered statistically significant $\left({ }^{*} P<0.05,{ }^{* *} P<0.01\right.$, $* * * P<0.001, * * * * P<0.0001)$. Evaluation of survival patterns in tumor-bearing mice was performed using the Kaplan-Meier method, and results were ranked according to the Mantel-Cox log-rank test. $P<0.05$ was considered statistically significant. Survival was defined as mice with tumors $<2500 \mathrm{~cm}^{3}$. Detailed information of the statistical test and number of replicates used in each experiment are appropriately reported in figure legend. All findings reported were reproducible and data shown are representative of at least two independent experiments, as specified, with comparable results in each experiment. The investigators were not blinded to allocation during experiments and outcome assessment.

Reporting summary. Further information on research design is available in the Nature Research Reporting Summary linked to this article.

\section{Data availability}

The RNA-seq data used for correlation analysis and Kyn-degrading enzymes expression were publicly available [http://www.cbioportal.org/] and deposited in the National Center for Biotechnology Information (NCBI) databases under the following accession numbers: Gene Expression Omnibus (GEO): GSE91061; Sequence Read Archive (SRA): SRP094781; and BioProject: PRJNA356761. For gene-expression analysis of AHR-related genes across immune subtypes, the normalized RNAseq expression matrices for the TCGA generated by the Pan Cancer Atlas initiative were downloaded [https://gdc.cancer.gov/about-data/ publications/pancanatlas]. All other relevant data are available in the article, supplementary information, or from the corresponding authors upon reasonable request.
Received: 1 August 2019; Accepted: 14 July 2020;

Published online: 11 August 2020

\section{References}

1. Mellor, A. L. \& Munn, D. H. Creating immune privilege: active local suppression that benefits friends, but protects foes. Nat. Rev. Immunol. 8, 74-80 (2008).

2. Holmgaard, R. B. et al. Tumor-expressed IDO recruits and activates MDSCs in a Treg-dependent manner. Cell Rep. 13, 412-424 (2015).

3. Munn, D. H. et al. GCN2 kinase in T cells mediates proliferative arrest and anergy induction in response to indoleamine 2,3-dioxygenase. Immunity https://doi.org/10.1016/j.immuni.2005.03.013 (2005).

4. Opitz, C. A. et al. An endogenous tumour-promoting ligand of the human aryl hydrocarbon receptor. Nature 478, 197-203 (2011).

5. Holmgaard, R. B., Zamarin, D., Munn, D. H., Wolchok, J. D. \& Allison, J. P. Indoleamine 2,3-dioxygenase is a critical resistance mechanism in antitumor $\mathrm{T}$ cell immunotherapy targeting CTLA-4. J. Exp. Med. 210, 1389-1402 (2013).

6. Wirth, L. J. et al. IDO1 as a mechanism of adaptive immune resistance to antiPD1 monotherapy in HNSCC. J. Clin. Oncol. https://doi.org/10.1200/ jco.2017.35.15_suppl.6053 (2018).

7. Long, G. V. et al. Epacadostat (E) plus pembrolizumab (P) versus pembrolizumab alone in patients (pts) with unresectable or metastatic melanoma: Results of the phase 3 ECHO-301/KEYNOTE-252 study. J. Clin. Oncol. 36, 108 (2018).

8. Kimura, A. et al. Aryl hydrocarbon receptor in combination with Stat1 regulates LPS-induced inflammatory responses. J. Exp. Med. 206, 2027-2035 (2009).

9. Wang, C., Ye, Z., Kijlstra, A., Zhou, Y. \& Yang, P. Activation of the aryl hydrocarbon receptor affects activation and function of human monocytederived dendritic cells. Clin. Exp. Immunol. 177, 521-530 (2014).

10. Liu, Y. et al. Tumor-repopulating cells induce PD-1 Expression in CD8+ $\mathrm{T}$ cells by transferring Kynurenine and AhR activation. Cancer Cell. https:// doi.org/10.1016/j.ccell.2018.02.005 (2018).

11. Puccetti, P. et al. Accumulation of an endogenous tryptophan-derived metabolite in colorectal and breast cancers. PLOS ONE. https://doi.org/ 10.1371/journal.pone.0122046 (2015).

12. Ferns, D. M. et al. Indoleamine-2,3-dioxygenase (IDO) metabolic activity is detrimental for cervical cancer patient survival. Oncoimmunology. https://doi. org/10.4161/2162402X.2014.981457 (2015).

13. Botticelli, A. et al. Can IDO activity predict primary resistance to anti-PD-1 treatment in NSCLC? J. Transl. Med. https://doi.org/10.1186/s12967-0181595-3 (2018).

14. Triplett, T. A. et al. Reversal of indoleamine 2,3-dioxygenase-Mediated cancer immune suppression by systemic kynurenine depletion with a therapeutic enzyme. Nat. Biotechnol. https://doi.org/10.1038/nbt.4180 (2018).

15. Riaz, N. et al. Tumor and Microenvironment Evolution during Immunotherapy with Nivolumab. Cell. https://doi.org/10.1016/j.cell.2017.09.028 (2017).

16. Thorsson, V. et al. The immune landscape of cancer. Immunity. https://doi. org/10.1016/j.immuni.2018.03.023 (2018).

17. Jürgens, B., Hainz, U., Fuchs, D., Felzmann, T. \& Heitger, A. Interferon- $\gamma$ triggered indoleamine 2,3-dioxygenase competence in human monocytederived dendritic cells induces regulatory activity in allogeneic T cells. Blood. https://doi.org/10.1182/blood-2008-12-195073 (2009).

18. Tsai, M. J. et al. Aryl hydrocarbon receptor agonists upregulate VEGF secretion from bronchial epithelial cells. J. Mol. Med. 93, 1257-1269 (2015).

19. Budhu, S. et al. CD8+ T cell concentration determines their efficiency in killing cognate antigen-expressing syngeneic mammalian cells in vitro and in mouse tissues. J. Exp. Med. https://doi.org/10.1084/jem.20091279 (2010).

20. Hoves, S. et al. Rapid activation of tumor-associated macrophages boosts preexisting tumor immunity. J. Exp. Med. https://doi.org/10.1084/ jem.20171440 (2018).

21. Takenaka, M. C. et al. Control of tumor-associated macrophages and T cells in glioblastoma via AHR and CD39. Nat. Neurosci. https://doi.org/10.1038/ s41593-019-0370-y (2019).

22. Nguyen, N. T. et al. Aryl hydrocarbon receptor negatively regulates dendritic cell immunogenicity via a kynurenine-dependent mechanism. Proc. Natl Acad. Sci. USA. https://doi.org/10.1073/pnas.1014465107 (2010).

23. De Henau, O. et al. Overcoming resistance to checkpoint blockade therapy by targeting PI3K $\gamma$ in myeloid cells. Nature 539, 443-447 (2016).

24. Sharma, P., Hu-Lieskovan, S., Wargo, J. A. \& Ribas, A. Primary, adaptive, and acquired resistance to cancer immunotherapy. Cell. https://doi.org/10.1016/j. cell.2017.01.017 (2017).

25. Highfill, S. L. et al. Disruption of CXCR2-mediated MDSC tumor trafficking enhances anti-PD1 efficacy. Sci. Transl. Med. https://doi.org/10.1126/ scitranslmed.3007974 (2014). 
26. Ngiow, S. F. et al. A threshold level of intratumor CD8+ T-cell PD1 expression dictates therapeutic response to anti-PD1. Cancer Res. https://doi. org/10.1158/0008-5472.CAN-15-1082 (2015).

27. Singh, N. P. et al. Activation of aryl hydrocarbon receptor (AhR) leads to reciprocal epigenetic regulation of Foxp3 and IL-17 expression and amelioration of experimental colitis. PLoS ONE. https://doi.org/10.1371/ journal.pone.0023522 (2011).

28. Mezrich, J. D. et al. An Interaction between Kynurenine and the Aryl Hydrocarbon Receptor Can Generate Regulatory T Cells. J. Immunol. https:// doi.org/10.4049/jimmunol.0903670 (2010).

29. Liu, C. et al. Treg cells promote the SREBP1-dependent metabolic fitness of tumor-promoting macrophages via repression of CD8 $+\mathrm{T}$ cell-derived interferon- $\gamma$. immunity. https://doi.org/10.1016/j.immuni.2019.06.017 (2019).

30. Proto, J. D. et al. Regulatory T cells promote macrophage efferocytosis during inflammation resolution. Immunity 49, 666-677.e6 (2018).

31. Bianchi-Smiraglia, A. et al. Inhibition of the aryl hydrocarbon receptor/ polyamine biosynthesis axis suppresses multiple myeloma. J. Clin. Invest. 128, 4682-4696 (2018).

32. Yamada, T. et al. Constitutive aryl hydrocarbon receptor signaling constrains type I interferon-mediated antiviral innate defense. Nat. Immunol. https://doi. org/10.1038/ni.3422 (2016).

33. Huang, A. C. et al. T-cell invigoration to tumour burden ratio associated with anti-PD-1 response. Nature. https://doi.org/10.1038/nature22079 (2017).

34. Pilotte, L. et al. Reversal of tumoral immune resistance by inhibition of tryptophan 2,3-dioxygenase. Proc. Natl Acad. Sci. USA. https://doi.org/ 10.1073/pnas.1113873109 (2012).

35. Li, H. et al. The landscape of cancer cell line metabolism. Nat. Med. https:// doi.org/10.1038/s41591-019-0404-8 (2019).

36. Munn, D. H. et al. Inhibition of T cell proliferation by macrophage tryptophan catabolism. J. Exp. Med. https://doi.org/10.1084/jem.189.9.1363 (1999).

37. Hwu, P. et al. Indoleamine 2,3-dioxygenase production by human dendritic cells results in the inhibition of T cell proliferation. J. Immunol. https://doi. org/10.4049/jimmunol.164.7.3596 (2000).

38. $\mathrm{Li}, \mathrm{H}$. et al. Metabolomic adaptations and correlates of survival to immune checkpoint blockade. Nat. Commun. https://doi.org/10.1038/s41467-01912361-9 (2019).

39. Simpson, T. R. et al. Fc-dependent depletion of tumor-infiltrating regulatory $t$ cells co-defines the efficacy of anti-CTLA-4 therapy against melanoma. J. Exp. Med. 210, 1695-1710 (2013).

40. Fares, C. M., Van Allen, E. M., Drake, C. G., Allison, J. P. \& Hu-Lieskovan, S. Mechanisms of resistance to immune checkpoint blockade: why does checkpoint inhibitor immunotherapy not work for all patients? Am. Soc. Clin. Oncol. Educ. B. 147-164. https://doi.org/10.1200/edbk_240837 (2019).

41. Hugo, W. et al. Genomic and transcriptomic features of response to anti-PD-1 therapy in metastatic melanoma. Cell 165, 35-44 (2016).

42. Wheeler, M. A., Rothhammer, V. \& Quintana, F. J. Control of immunemediated pathology via the aryl hydrocarbon receptor. J. Biol. Chem. https:// doi.org/10.1074/jbc.R116.767723 (2017).

43. Gutiérrez-Vázquez, C. \& Quintana, F. J. Regulation of the immune response by the aryl hydrocarbon receptor. Immunity. https://doi.org/10.1016/j. immuni.2017.12.012 (2018).

44. Wang, G. Z. et al. The aryl hydrocarbon receptor mediates tobacco-induced PD-L1 expression and is associated with response to immunotherapy. Nat. Commun. https://doi.org/10.1038/s41467-019-08887-7 (2019).

45. Muranski, P. et al. Tumor-specific Th17-polarized cells eradicate large established melanoma. Blood 112, 362-373 (2008).

46. Overwijk, W. W. et al. Tumor regression and autoimmunity after reversal of a functionally tolerant state of self-reactive CD8+ T cells. J. Exp. Med. 198 569-580 (2003).

47. Li, X., Kostareli, E., Suffner, J., Garbi, N. \& Hämmerling, G. J. Efficient Treg depletion induces T-cell infiltration and rejection of large tumors. Eur. J. Immunol. 40, 3325-3335 (2010).

48. Rooijen, N. Van \& Sanders, A. Liposome mediated depletion of macrophages: mechanism of action, preparation of liposomes and applications. J. Immunol. Methods 174, 83-93 (1994).

49. P. Matheu, M., Sen, D., Cahalan, M. D. \& Parker, I. Generation of bone marrow derived murine dendritic cells for use in 2-photon imaging. J. Vis. Exp. https://doi.org/10.3791/773 (2008)

50. Gao, J. et al. Integrative analysis of complex cancer genomics and clinical profiles using the cBioPortal. Sci. Signal. 6, pll (2013).

51. Cerami, E. et al. The cBio cancer genomics portal: an open platform for exploring multidimensional cancer genomics data. Cancer Discov. 2, 401-404 (2012).

52. Creighton, C. J. et al. Comprehensivemolecular characterization of clear cell renal cell carcinoma. Nature 499, 43-49 (2013).

53. Cancer, $\mathrm{T}$. et al. Comprehensive molecular characterization of urothelial bladder carcinoma. Nature 507, 315-322 (2014).

\section{Acknowledgements}

We thank the Flow Cytometry Core Facility at Memorial Sloan Kettering Cancer Center for technical assistance. This research was funded in part through the NIH/NCI Cancer Center Support Grant P30 CA008748, NIH/NCI R01 CA056821, NIH/NCI R01 CA215136, the Swim Across America, Ludwig Institute for Cancer Research, Ludwig Center for Cancer Immunotherapy at Memorial Sloan Kettering, Cancer Research Institute, Parker Institute for Cancer Immunotherapy and Breast Cancer Research Foundation.

\section{Author contributions}

L.F.C. designed and performed experiments, analysed data and wrote the paper. S.B. and R.Z performed and analyzed flow cytometry experiments and functional assays. J.T., J.C., A.C.C., M.G.M., and K.M. contributed with efficacy studies with KYN-101 compound and discussing experiments. M.G., I.C., D.R., and L.M. were involved with bioinformatics analysis of transcriptomics data. C.L. and S.P. provided technical assistance. C.W. and D.Z. were involved in study design and data interpretation. T.M. and J.D.W. developed the concepts, discussed experiments and wrote the paper. All authors discussed the results and commented on the manuscript.

\section{Competing interests}

J.T., J.C., A.C., K.M., and M.M. are employed by Ikena Oncology. S.B. is inventor on patent applications related to work on Lag3 and TIM3. R.Z. is inventor on patent applications related to work on GITR, PD-1, and CTLA-4. R.Z. is consultant for Leap Therapeutics. D.Z. reports grants from Merck, and consulting fees from Merck, Synlogic Therapeutics, Trieza Therapeutics, and Tesaro. D.Z. is an inventor on a patent concerning the use of Newcastle Disease Virus for cancer therapy. T.M. is a consultant for Leap Therapeutics, Immunos Therapeutics and Pfizer, and co-founder of Imvaq therapeutics. T.M. has equity in Imvaq therapeutics. T.M. reports grants from Bristol Myers Squibb, Surface Oncology, Ikena Oncology (former Kyn Therapeutics), Infinity Pharmaceuticals, Peregrine Pharmeceuticals, Adaptive Biotechnologies, Leap Therapeutics and Aprea. T.M. is inventor on patent applications related to work on oncolytic viral therapy, alphavirus-based vaccines, neo-antigen modeling, CD40, GITR, OX40, PD-1, and CTLA-4. J.D.W. is a consultant for Adaptive Biotech, Advaxis, Amgen, Apricity, Array BioPharma, Ascentage Pharma, Astellas, Bayer, Beigene, Bristol Myers Squibb Celgene, Chugai, Elucida, Eli Lilly, F Star, Genentech, Imvaq, Janssen, Kleo Pharma, Linneaus, MedImmune, Merck Pharmaceuticals, Neon Therapuetics, Ono, Polaris Pharma, Polynoma, Psioxus, Puretech, Recepta, Trieza, Sellas Life Sciences, Serametrix Surface Oncology, and Syndax. J.D.W. reports grants from Bristol Myers Squibb, Medimmune, Merck Pharmaceuticals and Genentech and honorarium from Esanex. J.D.W. has equity in Potenza Therapeutics, Tizona Pharmaceuticals, Adaptive Biotechnologies, Elucida, Imvaq, Beigene, Trieza and Linneaus. J.D.W. is inventor on patent applications related to work on DNA vaccines in companion animals with cancer, assays for suppressive myeloid cells in blood, oncolytic viral therapy, alphavirus-based vaccines, neoantigen modeling, CD40, GITR, OX40, PD-1, and CTLA-4. The remaining authors declare no competing interests.

\section{Additional information}

Supplementary information is available for this paper at https://doi.org/10.1038/s41467 020-17750-z.

Correspondence and requests for materials should be addressed to T.M. or J.D.W.

Peer review information Nature Communications thanks the anonymous reviewers for their contribution to the peer review of this work.

Reprints and permission information is available at http://www.nature.com/reprints

Publisher's note Springer Nature remains neutral with regard to jurisdictional claims in published maps and institutional affiliations.

Open Access This article is licensed under a Creative Commons Attribution 4.0 International License, which permits use, sharing, adaptation, distribution and reproduction in any medium or format, as long as you give appropriate credit to the original author(s) and the source, provide a link to the Creative Commons license, and indicate if changes were made. The images or other third party material in this article are included in the article's Creative Commons license, unless indicated otherwise in a credit line to the material. If material is not included in the article's Creative Commons license and your intended use is not permitted by statutory regulation or exceeds the permitted use, you will need to obtain permission directly from the copyright holder. To view a copy of this license, visit http://creativecommons.org/ licenses/by/4.0/

(C) The Author(s) 2020 